\title{
A global overview of pleiotropy and genetic architecture in complex traits
}

Authors: Kyoko Watanabe ${ }^{1}$, Sven Stringer ${ }^{1}$, Oleksandr Frei ${ }^{2}$, Maša Umićević Mirkov ${ }^{1}$, Tinca J.C. Polderman ${ }^{1}$, Sophie van der Sluis ${ }^{1,3}$, Ole A. Andreassen ${ }^{2,4}$, Benjamin M. Neale ${ }^{5-7}$, Danielle Posthuma ${ }^{1,3 *}$

\section{Affiliations:}

1. Department of Complex Trait Genetics, Center for Neurogenomics and Cognitive Research, Neuroscience Campus Amsterdam, VU University Amsterdam, The Netherlands.

2. NORMENT, KG Jebsen Centre for Psychosis Research, Institute of Criminal Medicine, University of Oslo, Oslo, Norway

3. Department of Clinical Genetics, Section of Complex Trait Genetics, Neuroscience Campus Amsterdam, VU Medical Center, Amsterdam, the Netherlands.

4. Division of Mental health and addiction Oslo University hospital, Oslo, Norway

5. Program in Medical and Population Genetics, Broad Institute of MIT and Harvard, Cambridge, MA, USA

6. Analytic and Translational Genetics Unit, Department of Medicine, Massachusetts General Hospital, Boston, MA, USA

7. Stanley Center for Psychiatric Research, Broad Institute of MIT and Harvard, Cambridge, MA, USA

*Correspondence to: Danielle Posthuma, Department of Complex Trait Genetics, VU University, De Boelelaan 1085, 1081 HV, Amsterdam, The Netherlands. Phone: +3120 5982823, Fax: +31 20 5986926, Email: d.posthuma@vu.nl

Word count: Abstract 181 words, Main text 5,762 words and Methods 4,572 words

References: 40

Display items: 4 figures and 2 tables

Extended Data: 11 figures

Supplementary Information: Text 3,401 words and 25 tables 


\section{ABSTRACT}

3 After a decade of genome-wide association studies (GWASs), fundamental questions in

4 human genetics are still unanswered, such as the extent of pleiotropy across the genome, the

5 nature of trait-associated genetic variants and the disparate genetic architecture across human

6 traits. The current availability of hundreds of GWAS results provide the unique opportunity

7 to gain insight into these questions. In this study, we harmonized and systematically analysed

84,155 publicly available GWASs. For a subset of well-powered GWAS on 558 unique traits,

9 we provide an extensive overview of pleiotropy and genetic architecture. We show that trait

10 associated loci cover more than half of the genome, and $90 \%$ of those loci are associated with

11 multiple trait domains. We further show that potential causal genetic variants are enriched in

12 coding and flanking regions, as well as in regulatory elements, and how trait-polygenicity is

13 related to an estimate of the required sample size to detect $90 \%$ of causal genetic variants.

14 Our results provide novel insights into how genetic variation contributes to trait variation. All

15 GWAS results can be queried and visualized at the GWAS ATLAS resource

16 (http://atlas.ctglab.nl). 


\section{MAIN TEXT}

18 Since the first genome-wide association study (GWAS) on macular degeneration in $2005^{1}$, over 3,000 GWASs have been published, for more than 1,000 traits, reporting on over tens of thousands of significantly associated genetic variants ${ }^{2}$. Results from GWASs have increased our insight into the genetic architectures of investigated traits, and for some traits, GWAS results have led to further insight into disease mechanisms ${ }^{3,4}$, such as autophagy for Crohn's disease $^{5}$, immunodeficiency for Rheumatoid arthritis ${ }^{6}$ and transcriptome regulation through FOXA2 in the pancreatic islet and liver for Type 2 diabetes $^{7}$. The emerging picture after over a decade of GWASs is that the majority of studied traits are highly polygenic and thus influenced by many genetic variants each of small effect $^{4,8}$, with disparate genetic architectures across traits ${ }^{9}$. Fundamental questions, such as whether all genetic variants or all genes in the human genome are associated with at least one trait, with many or even all traits, and whether the polygenic effects for specific traits are functionally clustered or whether they are randomly spread across the genome, are however still unanswered ${ }^{4,10,11}$. Answers to these questions would greatly enhance our understanding of how genetic variation leads to trait variation and trait correlation. Whereas GWAS primarily aims to discover genetic variants associated with specific traits, the current availability of a vast amount of GWAS results can be used to investigate some of these fundamental questions.

To this end, we compiled a catalogue of 4,155 GWAS results across 2,965 unique traits from 295 studies, including publicly available GWASs and new results for 600 traits from the UK

37 Biobank (http://atlas.ctglab.nl). These GWAS results were used in the current study to achieve the following aims; i) charting the extent of pleiotropy at trait-associated locus, gene, SNP and gene-set levels, $i i$ ) characterizing the nature of trait-associated variants (i.e. the distribution of effect size, minor allele frequency and biological functionality of traitassociated or credible SNPs), and iii) understanding the nature of the genetic architecture 
across a variety of traits and domains in terms of SNP heritability and trait polygenicity (see

Extended Data Fig. 1).

\section{Catalogue of 4,155 GWAS summary statistics for 2,965 unique traits}

We collected publicly available full GWAS summary statistics (last update $23^{\text {rd }}$ October 2018; see Methods). This resulted in 3,555 GWAS summary statistics from 294 studies. We additionally performed GWAS on 600 traits available from the UK Biobank release 2 cohort $\left(\mathrm{UKB} 2\right.$; release May 2017) ${ }^{12}$, by selecting non-binary traits with $>50,000$ European individuals with non-missing phenotypes, and binary traits for which the number of available cases and controls were each $>10,000$ and total sample size was $>50,000$ (see Methods,

Supplementary Information 1 and Supplementary Table 1-2). In total, we collected 4,155 based on previous studies ${ }^{13,14}$. The average sample size across curated GWASs was 56,250 subjects. The maximum sample size was 898,130 subjects for a Type 2 Diabetes metaanalysis $^{15}$. The 4,155 GWAS results are made available in an online database (http://atlas.ctglab.nl). The database provides a variety of information per trait, including SNP-based and gene-based Manhattan plots, gene-set analyses ${ }^{16}$, SNP heritability estimates $^{17}$, genetic correlations, cross GWAS comparisons and phenome-wide plots.

61 For the present study, we restricted our analyses to reasonably powered GWASs (i.e. sample size $>50,000$ ), to avoid including SNP effect estimates with relatively large standard errors (see Methods). By selecting a GWAS with the largest sample size per trait, it resulted in 558 GWASs for 558 unique traits across 24 trait domains. The average sample size of these 558 GWASs was 256,276, and 478 GWASs (85.7\%) were based on the UKB2 including 11 metaanalyses with UKB2, $46(8.2 \%)$ on the UK Biobank release 1 cohort (UKB1) including 8 
meta-analyses with UKB1, and the remaining were non-UKB cohorts. All results presented hereafter concern these selected 558 GWASs unless specified otherwise. The online database, however, allows researchers to reproduce similar analyses with custom selections of GWASs.

\section{The extent of pleiotropy}

Results of previous GWASs have shown significant associations of thousands of genomic loci with a large number of traits ${ }^{2,4}$. Given a finite number of segregating variants on the human genome, this suggests the presence of widespread pleiotropy. Pleiotropy may be informative to the reasons of co-morbidity between traits, as it may indicate an underlying shared genetic mechanism, and may aid in resolving questions regarding causal effects of one trait on another. However, the exact extent of pleiotropy across the genome is currently unknown ${ }^{4}$. We therefore investigated pleiotropy at locus, gene, SNP and gene-set levels. We defined pleiotropy as the presence of statistically significant associations with more than one trait domain as traits within domain tend to show stronger phenotypic correlations than between domains (see Supplementary Information 2 and Extended Data Fig. 2). Our definition thus refers to 'statistical pleiotropy', and includes situations of true pleiotropy (e.g. one SNP directly influences multiple traits), or situations where statistical associations to multiple traits are induced via causational effects of one trait on another, via phenotypic correlations between traits, or via a third common factor ${ }^{18}$. We defined the level of pleiotropy by the number of associated domains, and further grouped into four categories; multi-domain

87 (associated with traits from multiple domains), domain-specific (associated with multiple traits from a single domain), trait-specific (associated with a single trait) and non-associated (Methods). We then assessed whether pleiotropic associations at the locus, gene, SNP or gene set level are structurally or functionally different from trait- or domain-specific associations or non-associated sites. 
Pleiotropic genomic loci

94 The 558 GWASs yielded 41,511 trait-associated loci (from 470 traits, as 88 traits did not yield any genome-wide significant association after QC; see Methods). After grouping physically overlapping trait-associated loci, we obtained 3,362 grouped loci (Methods,

Extended Data Fig. 3, and Supplementary Table 4). The total summed length of these loci $(1706.0 \mathrm{Mb})$ covered $61.0 \%$ of the genome. Of these, $93.3 \%$ were associated with more than one trait and 90.0\% were multi-domain loci (Table 1 and Extended Data Fig. 4a, b). The multi-domain and domain-specific loci showed a significantly higher density of protein coding genes compared to non-associated genomic regions $(p=5.3 \mathrm{e}-16$ and $p=2.6 \mathrm{e}-4$; Fig. 1a and Supplementary Table 5).

The locus associated with the largest number of traits and domains (i.e. the most pleiotropic locus) was the MHC region (chr 6:25Mb-37Mb), which contained 441 trait-associated loci from 213 traits across 23 trait domains. The MHC region is well-known for its complex structure of linkage disequilibrium, spanning over 300 genes. The extremely pleiotropic nature of this region might, therefore, be explained by its long-ranged LD block due to overlap of multiple independent signals from multiple traits. Similarly, high locus pleiotropy, not limited to the MHC region, can occur purely due to the overlap of the LD blocks of the

110 loci in the grouped locus, and they may not share the same causal SNPs. By performing

111 colocalization (i.e. statistically identifying loci sharing the same causal SNP) for all possible

112 pairs of physically overlapping trait-associated loci (see Methods, Supplementary

113 Information 3 and Extended Data Fig. 3), we indeed observed a decrease in the number of

114 associated traits and trait domains per group of colocalized loci compared to loci defined by

115 physical overlap (Extended Data Fig. 4 and Supplementary Table 6). In addition, loci

116 grouped based on physical overlap often contained multiple independent groups of 
117 colocalized loci (Supplementary Table 6). Therefore, physical overlap of trait-associated

118 loci does not necessary mean that the same causal SNPs are involved in the traits associated

119 with such a grouped locus. Examination of pleiotropy at the gene or SNP level will provide

120 further insight into the nature of the pleiotropy observed at the locus level.

Pleiotropic genes

123 We next investigated the extent of pleiotropy at the gene level. For this, we conducted a gene-based analysis on 17,444 protein-coding genes using MAGMA for each trait ${ }^{16}$

125 (Methods). Of the 558 traits, 516 yielded at least one significantly associated gene and

$126 \quad 11,443(65.6 \%)$ genes were significantly associated to at least one trait (Supplementary

127 Table 7). Of these, $81.0 \%$ were associated with more than one trait and $66.9 \%$ were

128 associated with traits from multiple domains (Table 1 and Extended Data Fig. 5a, b). We

129 found that genes associated with at least one trait are significantly longer than genes that are

130 not associated with any of the 558 tested traits $(p=2.1 \mathrm{e}-194, p=8.7 \mathrm{e}-12$ and $p=3.8 \mathrm{e}-29$ for

131 multi-domain, domain-specific and trait-specific genes, respectively; Fig. 1b and

132 Supplementary Table 8). As the MAGMA algorithm is insensitive to bias caused by gene-

133 length, these findings are unlikely to be due to larger genes having an increased statistical

134 probability to be significantly associated (Supplementary Information 4, Extended Data

135 Fig. 5c and Supplementary Table 9). The multi-domain genes showed a significantly higher

136 probability of being intolerant to loss of function mutations ( $\mathrm{pLI}$ score) ${ }^{19}$ compared to trait-

137 /domain-specific and non-associated genes $(p=1.2 \mathrm{e}-79, p=4.8 \mathrm{e}-22$ and $p=2.8 \mathrm{e}-19$,

138 respectively; Fig. 1c and Supplementary Table 10), suggesting that more pleiotropic genes

139 are on average less tolerant to loss of function variants. The most pleiotropic genes are

140 located in the MHC region, yet a region on chromosome 3 also spanned multiple genes with 
high levels of pleiotropy (Extended Data Fig. 5a). In this region, $B S N$ was associated with

142 the largest number of trait domains (94 traits across 17 domains).

143 We next tested whether tissue specificity of genes was related to the level of pleiotropy by

144 counting the number of active tissues per gene based on gene expression profiles for 53 tissue

145 types obtained from GTEx ${ }^{20}$ (see Methods). The results showed that the proportion of genes

146 expressed in all 53 tissue types increases along with the level of pleiotropy $(p=9.7 \mathrm{e}-05$, Fig.

147 1d and Supplementary Table 11). This indicates that more pleiotropic genes tend to be

148 active in multiple tissue types, suggesting that those genes are involved in general biological

149 functions across the human body.

Pleiotropic SNPS

152 The level of pleiotropy at a locus or gene level does not necessarily translate to pleiotropy at

153 the level of the SNP. For example, within the same locus or gene, multiple SNPs may be

154 significantly associated with different traits. A locus or gene can thus show a higher level of

155 pleiotropy compared to individual SNPs. We, therefore, investigated the extent of pleiotropy

156 at the level of the SNP. To do so, we extracted 1,740,179 SNPs that were present in all 558

157 GWAS results. We first confirmed that this selection of SNPs had the same distribution of

158 their location across the genome and their functional consequences as all known SNPs on the

159 genome (Methods and Extended Data Fig. 6a, b). We note that some of the observed SNP-

160 pleiotropy may still be induced by LD, e.g. a SNP could reach genome-wide significance

161 because of its strong LD with a causal SNP. However, the purpose of this analysis is to

162 identify individual SNPs (not loci) that are associated with multiple trait domains and their

163 functions. Of these, 237,120 (13.6\%) were genome-wide significant $(p<5 \mathrm{e}-8)$ in at least one

164 of the 558 traits (Extended Data Fig. 6c and Supplementary Table 12). Out of 237,120

165 SNPs that were associated with at least one trait, $60.2 \%$ were associated with more than one 
167 Fig. 6d).

168 These pleiotropic SNPs spread broadly across the genome but were not evenly distributed,

169 i.e. chromosome 1, 11, 12, 15, 17, 20 and 22 showed relative enrichment of pleiotropic SNPs

170 (Supplementary Information 5 and Supplementary Table 13). Of all associated SNPs, the most pleiotropic SNP, located in the MHC region (rs707939; an intronic SNP of MSH5) was associated with 48 traits from 13 domains. There were 45 SNPs associated with 12 trait domains, of which 35 were located on chromosome $3,49.8 \mathrm{Mb}-50.1 \mathrm{Mb}$ overlapping with 5 protein coding genes, TRAIP, CAMKV, MST1R, MON1A and RBM6. These SNPs include two exonic SNPs, rs2681781 (synonymous on $C A M K V$ ) and rs2230590 (nonsynonymous on MST1R; Supplementary Table 12).

To investigate whether SNPs with a higher level of pleiotropy have different functional annotations than less pleiotropic SNPs, we investigated how functional consequence and tissue specificity in terms of expression quantitative trait loci (eQTLs) were represented

180 across different levels of SNP pleiotropy (Methods). We found that the proportion of intronic and exonic SNPs increased as a function of the level of pleiotropy ( $p=2.2 \mathrm{e}-3$ and $p=1.7 \mathrm{e}-2$, respectively); the proportion of exonic SNPs increased from less than $1 \%$ to over $5 \%$, and the proportion of intronic SNPs increased from less than $40 \%$ to over $50 \%$ (Fig. 1e and within flanking regions such as 5' and 3' untranslated regions (UTR) also increased with the number of associated domains. At the same time, we observed a steep decrease of the proportion of intergenic SNPs with increasing level of SNP pleiotropy ( $p=8.1$ e-4; Fig. 1e and Supplementary Table 14). Based on active eQTLs, the proportion of SNPs being eQTLs in a greater number of tissue types ( $>24$ tissue types out of 48 ) increased along with the number 
between 37 and 48 tissues, respectively) while SNPs in genes expressed in a single or less

193 Table 15). These results suggest that highly pleiotropic SNPs are more likely to be genic

194 (exonic and intronic) and less likely to be tissue specific.

Pleiotropic gene-sets

197 Pleiotropy at the level of trait-associated loci, genes or SNPs do not necessarily suggest the

198 presence of shared biological pathways across multiple traits. To assess the level of

199 pleiotropy at the level of gene-sets, reflecting a biological meaningful grouping of genes, we

200 performed MAGMA gene-set analyses for 558 traits using 10,650 gene-sets (Methods). In

201 total, $235(42.1 \%)$ traits showed significant association with one of 1,106 (10.4\%) gene-sets.

202 The most pleiotropic gene-set was 'Regulation of transcription from RNA polymerase II

203 promoter' (GO biological process) associated with 61 traits from 9 domains, followed by 7

204 other gene-sets associated with 7 domains, of which 5 of them were also involved in

205 regulation of transcription (Supplementary Table 16). We observed that the number of

206 genes in a gene-set was significantly larger for highly pleiotropic gene-sets (associated with more than one domain) compared to other gene-sets (domain-specific, trait-specific and nonassociated; $p=4.1 \mathrm{e}-12, p=1.6 \mathrm{e}-13$ and $p=1.2 \mathrm{e}-29$, respectively; Extended Data Fig. 7a, and

Supplementary Table 17). Since GO terms (55.6\% of tested gene-sets) have a hierarchical

210 structure, the larger gene-sets are more likely to be located at the top of the hierarchy,

211 representing more general functional categories.

212 In contrast to the pleiotropy at gene level where $80.9 \%$ genes were associated with more than

213 one trait, we only found $54.8 \%$ of the associated gene-sets to be pleiotropic (Table 1). We

214 observed that the proportion of pleiotropic genes per gene-set is not uniformly distributed,

215 and pleiotropic genes tend to cluster into a subset of gene-sets, explaining the decreased 
proportion of pleiotropic gene-sets compared to pleiotropic genes (Extended Data Fig. 7b,

217 c). At the same time, the higher proportion of trait-specific gene-sets $(45.2 \%)$ compared to

218 trait-specific genes (19.2\%) suggests that, given current definitions of gene-sets, the

219 combination of associated genes is rather unique to a trait and focusing on gene-sets to gain

220 insight into trait-specific biological mechanisms may be more informative than focusing on single genes (Supplementary Information 6).

\section{Genetic correlations across traits}

224 Above we showed that of all trait-associated loci, genes and SNPs that are associated with at

225 least one trait, $90.0 \%, 66.9 \%$ and $32.6 \%$ are associated with more than one domain,

respectively. Such wide-spread pleiotropy indices non-zero genetic correlations between

traits. To test whether genetic correlations are evenly present across traits or cluster into trait

domains, we computed pairwise genetic correlations $\left(r_{g}\right)$ across 558 traits using $\operatorname{LDSC}^{17}$.

We calculated the proportion of trait pairs with an $r_{g}$ that is significantly different from zero across all 558 traits, within domains and between domains. Out of 155,403 possible pairs across 558 traits, 24,106 pairs $(15.5 \%)$ showed significant genetic correlations after

Bonferroni correction $(p<0.05 / 155,403=3.2 \mathrm{e}-7)$ with an average $\left|r_{g}\right|$ of 0.38 .

In principle, if the trait domains contain traits that are biologically related, we would expect especially high in cognitive, 'ear, nose, throat', metabolic and respiratory domains, and for most of domains, average $\left|r_{g}\right|$ across significant trait pairs was higher than 0.38 (across all 
241 (51,750 with $\left.h^{2}{ }_{S N P}=0.0704\right)$ required $r_{g}$ to be above 0.39 or 0.18 , respectively, to gain a

242 power of 0.8 (Methods). Within domain, the majority of significant genetic correlations was

243 positive and the average $\left|r_{g}\right|$ was above 0.5 in most of the domains (Fig. 2a and

244 Supplementary Table 18). Between domains, the proportion of pairs with significant genetic

245 correlations was generally lower than within domains, and most of the domain pairs showed

246 average $\left|r_{g}\right|<0.4$ (Fig. 2b and Supplementary Table 19). Some trait domains showed a

247 predominance of negative genetic correlations with other domains, i.e. activity, cognitive,

248 reproduction and social interaction domains. We further clustered traits based on genetic

249 correlations, which resulted in the majority of clusters contained traits from multiple domains

250 (Methods, Supplementary Information 7 and Extended Data Fig. 8). These results

251 suggest that although $\left|r_{g}\right|$ is higher within domain than across domains, the trait domains do not necessary reflect genetic similarity across traits.

\section{The nature of trait-associated variants}

255 We now address the question whether trait-associated variants differ from genetic variants

256 that are not associated with any trait. For this purpose, we extracted all lead SNPs from each of the 558 GWASs. Lead SNPs were defined per trait at the standard threshold for genome- 
SNPs with a MAF above 0.01 was nearly uniform (Fig. 3a).

270 To gain insight into the distribution of effect sizes across lead SNPs, we calculated the standardized effect size $(\beta)$ from Z-statistics as a function of MAF and sample size ${ }^{21}$, and inspected the distribution of the squared standardized effect sizes $\left(\beta^{2}\right)$ for lead SNPs across all traits (Methods). $\beta$ ranged between 0.01 and 1.70, and $\beta^{2}$ is proportional to the variance explained. The median $\beta^{2}$ of the lead SNPs across all traits was 5.7e-4 (4.9e-4 and 6.0e-2 for lead SNPs with MAF $\geq 0.01$ and $<0.01$, respectively), and $94.6 \%$ of lead SNPs had a $\beta^{2}$ below 0.05 (Fig. 3b). Thus, the vast majority of lead SNPs thus explained less than $0.05 \%$ of the trait variance. We observed a relationship between MAF and standardized effect size, with rare variants $(\mathrm{MAF}<0.01)$ showing larger effect sizes $(\mathbf{F i g} .3 \mathbf{c})$. This is in line with the notion that rare variants are more likely to have large effects compared to common variants, as they are less likely to be under strong selective pressure ${ }^{22}$. However, we also note that statistical power for detecting the rare variants is un-stable ${ }^{23}$. Given that the proportion of rare lead SNPs is larger than the proportions in other MAF bins, it is possible that the distribution of the effect sizes has longer tails for SNPs with $\mathrm{MAF}<0.01$. For most of the traits, a similar relationship between MAF and standardized effect size was observed (Extended Data Fig.

285 9), but large variation across traits was seen in terms of the number of rare lead SNPs, with e.g. a large proportion of rare variants influencing nutritional and connective tissue domains 21). 
291 Here we sought to characterise differences in the distribution of functional annotations when comparing SNPs within trait-associated loci to all SNPs in the genome, and comparing lead

293 SNPs to SNPs in the trait-association loci (Methods). We first compared SNPs in the trait-

294 associated loci against the entire genome. The strongest enrichment of SNPs in trait

295 associated loci was seen in flanking regions (upstream, downstream, 5' and 3' UTR) with

296 average fold enrichment $(E) 1.31$ (Fig. 3d and Table 2). Non-coding SNPs, in total, covered

$29793.1 \%$ of SNPs in the trait-associated loci, while intergenic SNPs were significantly depleted

298 ( $E=0.83)$ and intronic SNPs significantly enriched compared to all SNPs in the genome

299 ( $E=1.17$; Table 2). SNPs in trait-associated loci were also slightly enriched for being exonic

300 compared to the entire genome $(E=1.07)$. Active chromatin states and eQTLs were also

301 significantly enriched with notably high enrichment of eQTLs ( $E=1.61$ and 5.95,

302 respectively; Table 2).

303 We next compared lead SNPs with SNPs in the trait-associated loci. The strongest

304 enrichment for lead SNPs was seen in exonic SNPs $(E=2.84)$ followed by flanking regions

305 ( $E=1.38)$, while intronic and intergenic regions were slightly depleted (average $E=0.95$; Fig.

306 3d and Table 2). These results clearly indicate that SNPs located in exonic and flanking

307 regions tend to show stronger effect sizes than other SNPs in the trait-associated loci. On the

308 other hand, active chromatin states showed slight enrichment $(E=1.08)$ while eQTLs were

309 significantly depleted ( $E=0.80$; Fig. 3e-f and Table 2). This suggests that SNPs within the

310 trait-associated loci largely overlap with regulatory elements but these elements do not

311 always have the strongest effect sizes within the loci.

312

313 Characterization of credible set SNPs based on fine-mapping

314 Owing to the small effect sizes of variants in complex traits and extensive LD throughout the 315 human genome, there is a reasonable chance that lead SNPs (i.e. defined based on LD and P- 
values) are not the causal SNPs in the trait-associated loci ${ }^{24}$, even when the causal SNPs are

317 actually measured or imputed. Statistical fine-mapping utilizes evidence of the associations at

318 each variant in the loci (effect sizes and LD structure) to assign posterior probability of each

319 specific model at particular locus, which are then used to infer the posterior probabilities of

320 each SNP being included in the model (posterior inclusion probability, PIP) and ascertain the minimum set of SNPs required to capture the likely causal variant. We performed finemapping using FINEMAP software ${ }^{25}$ for each trait-associated locus, setting the maximum number of SNPs in the causal configuration $(k)$ to 10 and using randomly selected $100 \mathrm{k}$ individuals from UKB2 as a reference panel (see Methods). From all of the loci associated with at least one of the 558 traits, we obtained a list of credible SNPs with PIP $>0.95$ consists of 196,542 SNPs (Supplementary Information 9).

327 Next we characterized credible SNPs in respect to their functional annotations, similar as done above with lead SNPs. We thus compared SNPs in the fine-mapped regions to all SNPs in the genome, and credible SNPs to SNPs in the fine-mapped regions. The enrichment

330 pattern of SNPs in the fine-mapped regions was similar to SNPs in the trait-associated loci;

331 i.e. significant enrichment of SNPs in intronic and flanking regions but the fold enrichment was much smaller (Fig. 3d and Table 2). This is mainly because the fine-mapped regions are often larger than the trait-associated loci by taking $50 \mathrm{~kb}$ around the top SNPs of the traitassociated loci. In contrast, fold enrichment of exonic SNPs was slightly higher than traitassociated loci (Table 2). As we observed higher gene-density around the trait-associated

336 loci, expanding the loci resulted in larger proportion of exonic regions. Both active chromatin state and eQTLs were significantly enriched, however, fold enrichment of eQTLs was notably less than trait-associated loci (Fig. 3e-f and Table 2). Similar to the lead SNPs, credible SNPs showed strong enrichment in exonic $(E=1.40)$ and flanking regions $(E=1.29)$, 
state is consistent with the result observed in the lead SNPs $(E=1.51)$, eQTLs were also

\section{Table 2).}

344 In summary, the number of credible SNPs is 4.5 times larger than the number of lead SNPs,

345 since for determining lead SNPs, all SNPs that have high LD with lead SNPs are discarded

346 while the fine-mapping captures likely causal SNPs given the observed pattern of association

347 and LD structure. Lead SNPs and credible SNPs show different distributions of enrichment in

348 tested biological functions. We observed a decreased proportion of exonic SNPs and an

349 increased proportion of non-coding or regulatory SNPs within the credible SNPs compared to

350 the lead SNPs. These findings may be due to the fact that coding SNPs tend to have higher

351 effect sizes and are more often assigned as lead SNPs, while the fine-mapping in regions

352 containing some of these causal coding variants may disperse a proportion of probability to

353 adjacent variants. On the other hand, in loci where causal variants are acting through

354 regulatory mechanisms, the credible sets may be more likely to capture the actual, single or

355 multiple causal variants as compared to the lead SNPs.

357 The nature of genetic architecture

358 The genetic architecture of a trait reflects the characteristics of genetic variants that

359 contribute to the phenotypic variability, and is defined by e.g. the number of variants

360 affecting the trait, the distribution of effect sizes, the MAF and the level of interactions

361 between SNPs ${ }^{9}$. To gain insight into how the genetic architecture varies across multiple

362 complex traits, we assessed the SNP heritability $\left(h^{2}{ }_{S N P}\right)$ and the polygenicity of 558 traits. 
$h^{2}{ }_{S N P}$ is an indication of the total amount of variance that is captured by the additive effects of all variants included in a GWAS. $h^{2}{ }_{S N P}$ depends on several factors, such as the number of

367 SNPs included in the analyses based on their MAF given the current sample size, the polygenicity of the trait (i.e. how many SNPs have an effect) and the distribution of effect sizes. We estimated $h^{2} S N P$ for each trait using $\operatorname{LDSC}^{17}$ and SumHer from LDAK ${ }^{26,27}$ (Methods). The estimates of $h^{2}{ }_{S N P}$ using LDSC and SumHer showed strong positive correlation ( $r=0.77$ and $p=3.8 \mathrm{e}-111$; Fig. 4a). Therefore, we focus on estimates based on LDSC, hereafter, however complete results are available in Supplementary Table 22 and discussed in Supplementary Information 10 (Extended Data Fig. 11). The highest $h^{2}{ }_{S N P}$ was observed for height $\left(h^{2}{ }_{S N P}=0.31\right)$ followed by bone mineral density $\left(h^{2}{ }_{S N P}=0.27\right)$. Of 558 traits, 214 traits, with an average sample size 292,267, showed $h^{2}{ }_{S N P}$ less than 0.05 . Most of these traits are classically regarded as 'environmental' (e.g. current employment status, illness of family members and transport types or activity traits including frequency and type of physical activities and type of accommodation), and tend to have a low $H^{214}$. For these traits, the number of detected trait-associated loci is also very low with a median 3 . Given the combination of current sample size of $>200,000$ and low $h^{2}{ }_{S N P}$, this suggests that for these traits increasing the sample size may not lead to a substantial increase in detected loci.

384 The general observation from GWASs is that with increasing sample size, detected signals

385 become not only more reliable but also more numerous, as with increasing power, smaller

386 SNP effects may be detected. The total number of associated SNPs, the amount of variance

387 they collectively represent, the distribution of effect sizes across the associated SNPs and how many additional individuals are expected to be needed for the detection of a fixed 
number of novel SNPs, are indications of the polygenicity of a trait. Such polygenicity may vary across traits, and can be informative for designing SNP-discovery studies.

391 To obtain an indication of trait-polygenicity, we applied the Causal Mixture Model for

392 GWAS summary statistics (MiXeR) ${ }^{28}$ to estimate $\pi$ (fraction of independent causal SNPs, 393 polygenicity) and $\sigma_{\beta}^{2}$ (variance of effect sizes of the causal SNPs, discoverability; see

394 Methods). $\pi$ ranges between 0 and 1, and a high $\pi$ indicates a high level of polygenicity, while a high $\sigma_{\beta}^{2}$ indicates a high level of discoverability of causal SNPs for the traits. Since the standard error of the model estimates become larger for traits with very small $h^{2}$ SNP due to the small effect sizes, we only discuss the results of 197 out of 558 traits with $h^{2}{ }_{S N P}>0.05$ and standard error of $\pi$ less than $50 \%$ of the estimated value (as recommended by O. Frei; full results are available in Supplementary Table 23). We observed, as expected, a negative relationship between polygenicity and discoverability ( $r=-0.89$ and $p=4.93 \mathrm{e}-70)$, confirming that highly polygenic traits tend to have less causal SNPs with larger effect sizes (Fig. 4b). The majority of traits (i.e. 116 traits) showed high polygenicity with $\pi>1 \mathrm{e}-3$ (more than $0.1 \%$ of all SNPs are causal). The highest polygenicity was observed in Major depressive disorder with $0.6 \%$ of SNPs being causal, while some traits, such as fasting glucose and serum urate level showed relatively low polygenicity (Fig. $4 \mathbf{b}$ and Supplementary Table 23). The traits with polygenicity $>0.1 \%$ showed, on average, 8 times less discoverability compared to other traits with $<0.1 \%$ of causal SNPs. The GWAS discoveries for traits with lower polygenicity and high discoverability will saturate with a lower sample size compared to the traits with

409 higher polygenicity. Indeed, the estimated sample size, which is required to explain $90 \%$ of

410 SNP heritability by genome-wide significant SNPs, is positively correlated with polygenicity

$411 \quad(r=0.84$ and $p=6.30 \mathrm{e}-54)$, and extremely polygenic traits require tens of millions of subjects

412 to identify $90 \%$ of causal SNPs at a genome-wide significant level (Fig. $4 \mathbf{c})$. 


\section{Discussion}

415 The availability of hundreds of GWAS results provides the unique opportunity to gain insight

416 into currently understudied questions regarding the genetic architecture of human traits. To

417 facilitate such insight, we compiled a catalogue of 4,155 GWASs which can be queried

418 online (http://atlas.ctglab.nl). We selected 558 well-powered GWASs to answer fundamental

419 questions concerning the extent of pleiotropy of loci, genes, SNPs and gene-sets,

420 characteristics of trait-associated variants and the polygenicity of traits.

421 We found that the total summed length of trait-associated loci for the 558 analysed traits

422 covered more than half $(60.1 \%)$ of the genome. $90 \%$ of the grouped loci contained

423 associations with multiple traits across multiple trait domains. High locus pleiotropy can

424 occur in two scenarios; $i$ ) when the same gene in a locus is associated with multiple traits or

425 ii) when different genes or SNPs in the same locus are associated with multiple traits but due

to LD the same locus is indicated. Our results showed that the proportion of pleiotropic

associations dropped from $90 \%$ at the locus level to $63 \%$ at the gene level, and to $31 \%$ at the

SNP level. These results show that although locus pleiotropy is widespread, pleiotropy at the

429 level of genes and SNPs is much less abundant. This suggests that a gene can be involved in

430 two distinct traits but how that gene is affected by the causal SNPs might differ. For instance,

431 the function of the gene can be disrupted through a coding SNP for one trait, but expression

432 of the same gene can be affected through a regulatory SNP for another trait.

433 Genes and SNPs that had a higher level of pleiotropy, were less tissue specific in terms of

434 gene expression and active eQTLs. This suggests that SNPs and genes associated with

435 multiple trait domains are more likely to be involved in general biological functions. Indeed,

436 the top highly pleiotropic gene-sets were mostly involved in regulation of transcription which

437 is an essential biological mechanism for any kind of cell to be functioning. Highly pleiotropic

438 genes, therefore, can explain general vulnerability to a wide variety of traits, yet they may be 
less informative when the aim is to understand the causes of a specific trait. Although a large

440 proportion of trait-associated genes are pleiotropic, the majority of trait associated gene-sets

441 were trait-specific. Thus, the trait-specific combination of genes is highly informative, and

442 future studies aimed at improved annotation of gene-functions will be needed to understand

443 trait-specific gene association patterns.

444 It has been widely acknowledged that almost $90 \%$ of GWAS findings fall into non-coding

445 regions $^{2}$. Our results indeed show that $89.1 \%$ of the lead SNPs are non-coding, including

446 intergenic (34.3\%) and intronic (43.6\%) SNPs. similarly, of the credible SNPs 92.4\% were

447 non-coding (intergenic $33.4 \%$ and intronic $48.1 \%$ ). However, we showed different patterns

448 when considering lead and credible SNPs; intergenic SNPs were depleted and the intronic

449 SNPs were enriched in both the lead and credible SNPs. We also observed strong enrichment

450 of the lead and credible SNPs in coding and flanking regions. These results indicate that both

451 SNPs with the largest effect size (the lead SNPs) and the most likely causal SNPs (credible

452 SNPs) within a locus tend to be located within or close to the genes. Although active

453 chromatin states were enriched in both lead and credible SNPs, eQTLs were only enriched in

454 credible SNPs but depleted in lead SNPs. This implies that likely causal regulatory SNPs do

455 not necessarily have the strongest effect sizes in a locus.

456 Our analyses showed that the majority of analysed traits are highly polygenic with more than

$457 \quad 0.1 \%$ of SNPs being causal. For those highly polygenic traits, over 10 s of millions of

458 individuals are required to identify all SNPs at genome wide significance $(p<5 \mathrm{e}-8)$ that can

459 explain at least $90 \%$ of the phenotypic variance explained by additive genetic effects. In the

460 case of polygenic traits, individuals have almost unique combinations of risk/effect alleles for

461 a specific disease or trait. With higher levels of polygenicity, and thus larger quantities of

462 causal SNPs, the possible combinations of them also increase. This substantially increases the

463 degree of genetic heterogeneity of the trait, and complicates the detection of genetic effects as 
464 the effect sizes of individual SNPs that are yet to be detected are even smaller than those

465 observed in current GWASs.

466 In conclusion, our analyses have provided novel insight into the extent of pleiotropy, the

467 nature of associated genetic regions and how traits differ in genetic architectures. This

468 knowledge can guide the design of future genetic studies. 
METHODS

\section{Publicly available GWAS summary statistics}

471 GWAS summary statistics were curated from multiple resources and were included only

472 when the full set of SNPs were available. We excluded whole exome sequencing studies.

473 This yielded 2,288 GWASs from 33 consortia and any other resources where summary

statistics are available (last update $23^{\text {rd }}$ October 2018). From dbGAP, we obtained 2,659

unique datasets (ftp://ftp.ncbi.nlm.nih.gov/dbgap/Analyses_Table_of_Contents.txt, last

publication was available and sample size for a specific trait was explicitly mentioned in the original study. We excluded non-GWAS studies (e.g. PAGE (Prenatal Assessment of

Genomes and Exomes) studies) and GWASs with immune-chip, whole exome sequencing and replication cohorts (exact reasons of exclusion for each dataset is available in

Supplementary Table 24).

482 Together this resulted in a total of 3,555 GWAS summary statistics. The complete list and

483 detailed information for each GWAS with summary statistics is available in Supplementary

484 Table 3 (atlas ID 1-3184, 3785-4155).

\section{UK Biobank GWAS summary statistics}

487 Additional to the summary statistics available from external studies, we performed GWASs 488 of traits from UK Biobank release 2 cohort (UKB2) ${ }^{12}$ under application ID 16404. We only 489 used phenotype fields with first visit and first run (e.g. f.xxx.0.0) with exceptions for multicoded phenotypes, which allowed to assign more than one code for a single subject (see

491 Supplementary Information 1, 2). From the 1,940 unique field IDs to which we had access,

$492755 \mathrm{had}>50,000$ subjects with non-missing values. They are assigned to field name using 493 ukb_field.tsv obtained from http://biobank.ctsu.ox.ac.uk/crystal/download.cgi (last accessed 

status phenotypes (i.e. completion status, answered a specific question), ethnicity, genomic phenotypes and any other phenotypes that are not relevant for performing a GWAS. For each phenotype, we provided reason of exclusions in Supplementary Table 1. This resulted in 434 unique fields including 49 multi-coded phenotypes. 385 phenotypes were considered quantitative when the phenotype value was quantitative or categorical, and could be ordered.

502 Phenotypes coded by yes/no were considered as binary with a few exceptions

503 (Supplementary Table 1). For quantitative and binary phenotypes, subjects with phenotype codes -1 for "Do not know" or -3 for "Prefer to not answer" were excluded and the original phenotype code as described in the UK biobank data showcase was used unless specified in dichotomized each code to dummy binary phenotypes (cases for 1 and controls for 0 ) and included subjects with phenotype code -7 for "None of the above" as controls. Again, subjects with phenotype codes -1 for "Do not know" or -3 for "Prefer to not answer" were excluded. For example, field 670 based on UKB Data-Coding 100286 is coded from 1 to 5

511 and dichotomization results in five phenotypes such as 1 vs all others, 2 vs all others and so

512 on. Detailed definitions of multi-coded phenotypes are described in Supplementary Table 2.

513 After phenotyping, we selected phenotypes that had at least 50,000 European subjects. For

514 binary traits, we further restricted to traits with at least 10,000 cases and controls. This

515 resulted in a total of 600 traits ( 260 quantitative and 340 binary traits). Note that the final

516 total sample size encoded in the atlas database (http://atlas.ctglab.nl) might be less than

51750,000 due to lack of genotype data or missing values in covariates. 
518 GWAS was performed for up to $10,846,944$ SNPs with MAF $>0.0001$ using PLINK $2^{29}$,

519 while correcting for array, age (f.54.0.0), sex (f.31.0.0), Townsend deprivation index

520 (f.189.0.0), assessment centre (f.21003.0.0) and 20 PCs. Linear or logistic models were used

521 for quantitative or binary traits, respectively.

522 The complete list of traits from UK biobank release 2 analysed in this study is available in

523 Supplementary Table 3 (atlas ID 3185-3784).

525 Pre-processing of GWAS summary statistics

526 Curated summary statistics were pre-processed to standardize the format. SNPs with $\mathrm{p} \leq 0$ or

$527>1$, or non-numeric values such as "NA" were excluded. For summary statistics with non-

528 hg19 genome coordinates, liftOver software was used to align to hg19. When only rsID was

529 available in the summary statistics file without chromosome and position, genome

530 coordinates were extracted from dbSNP 146. When rsID was missing, it was assigned based

531 on dbSNP 146. When only the effect allele was reported, the other allele was extracted from

532 dbSNP 146.

\section{Definition of lead SNPs and trait-associated loci}

535 For each GWAS, we defined lead SNPs and genomic trait-associated loci as described before

$536{ }^{30}$. First, we defined independent significant SNPs with $p<5 \mathrm{e}-8$ and independent at $r^{2}<0.6$,

537 and defined LD blocks for each of independent significant SNPs based on SNPs with $\mathrm{p}<0.05$.

538 Of these SNPs, we further defined lead SNPs that are independent at $r^{2}<0.1$. We finally

539 defined genomic trait-associated loci by merging LD blocks closer than $250 \mathrm{~kb}$. Each trait-

540 associated locus was then represented by the top SNP (with the minimum P-value) and its

541 genomic region was defined by the minimum and maximum position of SNPs which are in 
$\mathrm{LD}\left(r^{2} \geq 0.6\right)$ with one of the independent significant SNPs within the (merged) locus. We

543 used 1000 genome phase $3(1000 \mathrm{G})^{31}$ as a reference panel to compute LD for most of the

544 GWASs in the database. For each GWAS, the matched population (from AFR, AMR, EAS,

545 EUR, SAS) was used as the reference based on the information obtained from the original

546 study. For trans-ethnic GWASs, the population with the largest total sample size was used.

547 When the GWAS was based on the UKB release 1 cohort (UKB1), we used 10,000 randomly

548 sampled unrelated White British subjects from UKB1 as reference. For other GWASs

549 performed in this study or GWASs based on the UKB2, 10,000 randomly selected unrelated

550 EUR subjects were used as a reference. Non-bi-allelic SNPs were excluded from any

551 analyses.

552 The reference panel used for each GWAS is provided in the column "Population" of

553 Supplementary Table 3. For trans-ethnic GWASs, the first population was used as

554 reference, e.g. EUR+EAS+SAS means EUR had the largest sample. GWASs based on the

555 UKB cohort was encoded either "UKB1 (EUR)" for UKB release 1 or "UKB2 (EUR)" for

$556 \quad$ UKB release 2.

MAGMA gene and gene-set analysis

559 We performed MAGMA v1.06 ${ }^{16}$ gene and gene-set analyses for every GWAS in the

560 database. For gene-analysis, 20,260 protein-coding genes were obtained using the $\mathrm{R}$ package

561 BioMart (Ensembl build v92 GCRh37). SNPs were assigned to genes with $1 \mathrm{~kb}$ window at

562 both sides. The reference panel of corresponding populations used for each GWAS was based

563 on either $1000 \mathrm{G}, \mathrm{UKB} 1$ or UKB2 as described in the previous section. The gene-set analysis

564 was performed with default parameters (snp-wise mean model). Gene-set analysis was

565 performed for 4,737 curated gene-sets (C2) and 5,917 GO terms (C5; 4,436 biological 
processes, 580 cellular components and 901 molecular functions) from MsigDB v6.1

(http://software.broadinstitute.org/gsea/msigdb, last accessed 20 Apr 2018) . $^{32}$

\section{SNP heritability and genetic correlation with LD score regression}

570 We performed LD score regression (LDSC) ${ }^{17}$ for each GWAS to obtain SNP heritability and pairwise genetic correlations. Pre-calculated LD scores for 1000G EUR and EAS populations were obtained from https://data.broadinstitute.org/alkesgroup/LDSCORE/ (last accessed 26 Nov 2016) and LD score regression was only performed for GWASs with either an EUR or EAS population and when the number of SNPs in the summary statistics file was $>450,000$. LDSR was performed only for HapMap3 SNPs excluding the MHC region (25Mb-34Mb). When the signed effect size or odds ratio was not available in the summary statistics file, "-a1-inc" flag was used. As recommended previously ${ }^{33}$, we excluded SNPs with chi-square $>80$. For binary traits, the population prevalence was curated from the literature (only for diseases whose prevalence was available, Supplementary Table 25) to compute SNP heritability at the liability scale with "--samp-prep" and "--pop-prep" flags. For most of the personality/activity (binary) traits from UKB2 cohort, we assumed that the sample prevalence is equal to the population prevalence since the UK Biobank is a population cohort and not designed to study a certain disease/traits. Likewise, when population prevalence was not available, sample prevalence was used as population prevalence for all other binary traits.

Genetic correlations were computed for pair-wise GWASs with the following criteria as suggested previously ${ }^{33}$ :

- GWASs of EUR population or more than $80 \%$ of samples are EUR.

- The number of SNPs $>450,000$ 
- $\quad$ SNP heritability Z score $>2$

592

In total, pairwise genetic correlations were computed for 1,090 GWASs in the database.

\section{Selection of GWASs for cross-phenotype analyses}

596 From the 4,155 curated GWASs in the database, we selected 558 GWASs with unique traits

597 for cross-phenotype analyses based on the following criteria.

- Minimum sample size 50,000 and both cases and controls are $>10,000$ for binary phenotypes.

600

- The number of SNPs in the summary statistics is $>450,000$.

- GWAS is based on EUR population or $>80 \%$ of the samples are EUR. If summary statistics of both trans-ethnic and EUR-only are available, use EUR-only GWAS.

- Exclude sex-specific GWAS, unless the phenotype under study is only available for a specific sex (e.g., age at menopause). If sex-specific and sex-combined GWASs are available, use sex-combined GWAS.

- Z-score of $h^{2}{ }_{S N P}$ computed by LDSC is $>2$

- Signed effect size (beta or odds ratio) is available in the summary statistics.

- Effect and non-effect alleles are explicitly mentioned in the header or elsewhere.

612 UKB2 GWASs performed in this study are further filtered based on the following:

- Exclude cancer screening or test phenotypes.

- Exclude phenotypes of parents' age and parents' still alive. 
- If exactly the same traits were diagnosed by an expert (e.g. doctor) and self-reported, use the expert qualification.

- If exactly the same traits were present as main and secondary diagnoses, both are included.

- Phenotypes with large extremes were excluded from the analyses when the difference between the maximum value and 99 percentiles of the standardized phenotype value is $>50$.

624 There was one exception for height GWAS, where a meta-analysis by Yengo et al. ${ }^{34}$ (ID 4044) has the larger sample size, however the meta-analysis was limited to $\sim 2.4$ million HapMap 2 SNPs. Since over 10 million SNPs are included in most of the selected GWASs, this smaller number of SNPs can bias our analyses. Therefore, the second largest GWAS (UKB2 GWAS performed in this study, ID 3187) was used instead. This resulted in total of 558 GWASs, across 24 domains, which were subsequently used in the cross-phenotype analyses in this study. These 558 GWASs are specified in Supplementary Table 3.

\section{Pleiotropic trait-associated loci}

633 To define pleiotropic loci for the 558 traits (GWASs), we first extracted trait-associated loci

634 on autosomal chromosomes. We excluded any locus with a single SNP (no other SNPs have $\left.635 r^{2}>0.6\right)$ as these loci are more likely to be false positives. We then grouped physically 636 overlapping loci across 558 traits. In a group of loci, it is not required that all individual trait637 associated loci are physically overlapping but merging them should result in a continuous 638 genomic region. For example, when trait-associated loci A and B physically overlap and trait639 associated loci B and C also physically overlap, but A and C do not, these three trait640 associated loci were grouped into a single group of loci (Extended Data Fig. 3). Therefore, a 641 grouped locus could contain more than one independent locus from a single trait when gaps 
between them were filled by loci from other traits. The grouped loci were further assigned to

643 three categories, $i$ ) multi-domain locus when a loci group contained traits from more than one

644 domain, $i$ ) domain specific locus when a loci group contained more than one trait from the

645 same domain, and iii) trait specific locus when a locus did not overlap with any other loci.

646 We compared the distribution of gene density across four association categories of the loci;

647 multi-domain, domain specific and trait specific loci, and non-associated genomic regions.

648 To define non-associated genomic regions, we extracted the minimum and maximum

649 positions that were covered by $1000 \mathrm{G}$, and the gap regions of grouped trait-associated loci

650 were defined as non-associated regions. The gene density was computed as a proportion of a

651 region that was overlapping with one of 20,260 protein-coding genes obtained from Ensembl

652 v92 GRCh37. We then performed pairwise Wilcoxon rank sum test (two sided).

653

\section{Colocalization of trait-associated loci}

655 To evaluate if physically overlapping trait-associated loci also share the same causal SNPs, 656 we performed colocalization using the coloc.abf (Approximate Bayes Factor colocalization 657 analysis) function of the coloc package in $\mathrm{R}^{35}$. Colocalization analysis was performed for all possible pairs of physically overlapping trait-associated loci across 558 traits. When two loci from different traits were physically overlapping but there were no SNPs that were present in both GWAS summary statistics in that overlapping region, colocalization was not performed.

661 The inputs of the coloc.abf function are P-value, MAF and sample size for each SNP. When

662 MAF was not available in the original summary statistics, it was extracted from the matched

663 reference panel. For binary traits, sample prevalence was additionally provided based on total cases and controls of the study.

665 The coloc. abf function assumes a single causal SNP for each trait and estimates the posterior 666 probability of the following 5 scenarios for each testing region; $H_{0}$ : neither trait has a genetic 
association, $H_{1}$ : only trait 1 has a genetic association, $H_{2}$ : only trait 2 has a genetic

668

669

670

671

672

673

674

675

676

678

680

681

682

683

684 association, $H_{3}$ : both trait 1 and 2 are associated but with different causal SNPs and $H_{4}$ : both trait 1 and 2 are associated with the same single causal SNP. In this study, as we pre-define the trait-associated loci for each trait which already discard scenarios $H_{0}$ to $H_{2}$, we are only interested whether $H_{4}$ is most likely. We therefore defined, a pair of loci as colocalised when the posterior probability of $H_{4}$ is $>0.9$. We note that it is possible that genomic regions outside of the pre-defined trait-associated loci can also colocalize with other traits. However, we limited the analyses to the pre-defined trait-associated loci in this study, to be consistent with the level of pleiotropy measured by physical overlap of the loci.

Within a grouped locus defined based on physical overlap (see above), we further grouped loci based on a colocalization pattern. To do so, we considered colocalization pattern across group of physically overlapping loci as a graph in which nodes represent trait-associated loci and edges represent colocalization of the loci First, loci which did not colocalized with any other loci were considered as independent loci. For the rest of the loci, we identified connected components of the graph (Extended Data Fig. 3). This does not require all loci within a component to be colocalized with each other. For example, when locus A is colocalized with locus $\mathrm{B}$, and locus B is colocalized with locus $\mathrm{C}$, but locus $\mathrm{A}$ is not colocalized with locus $\mathrm{C}$, all loci A, B and C are grouped into a single connected component. Detailed results are discussed in the Supplementary Information 3.

\section{Pleiotropic genes}

For gene level pleiotropy, we extracted MAGMA gene analysis results for the 558 traits where 17,444 genes on autosomal chromosomes were tested in all GWASs. For each trait, genes with $p<2.87 \mathrm{e}-6(0.05 / 17,444)$ were considered as significantly associated. We did not correct the P-value for testing 558 traits since our purpose is not to identify genes associated 
with one of the 558 traits but to evaluate the overlap of trait-associations (when GWAS was performed for a single trait) across the 558 traits, and this applies to SNPs and gene-set level pleiotropy. The trait associated genes were further categorized into three groups in a similar way as for trait-associated loci, i.e. $i$ ) multi-domain genes that were significantly associated with traits from more than one domain, ii) domain-specific genes that were significantly associated with more than one trait from the same domain and iii) trait-specific genes that were significantly associated with a single trait.

699 We compared gene length and pLI score across genes in three different association categories and non-associated genes. Gene length was based on the start and end position of genes extracted from the R package biomaRt and $\mathrm{pLI}$ score was obtained from rank sum tests for pLI scores (both two sided).

For each protein coding gene, we first assessed whether a gene is expressed or not in each of 53 tissue types based on expression profile obtained from GTEx v $7^{20}$. We defined genes as expressed in a given tissue type if the average TPM is $>1$. For each of 17,444 genes, we then counted the number of tissue types where the gene is expressed and grouped them into six categories, i.e. genes expressed in $i$ ) a single tissue type (tissue specific genes), $i$ ) between 2 and 13, iii) between 14 and 26, ix) between 27 and 39, x) between 40 and 52, and $x i$ ) 53 (all)

711 tissue types. At each number of associated domains (from 1 to 10 or more domains), we re-

712 calculated the proportion of genes in each of the 6 categories, and performed the Fisher's exact tests (one-sided) against baseline (the proportion relative to all 17,444 genes) to

714 evaluate if the proportion is higher than expected. 
717 We extracted 1,740,179 SNPs that were present in all 558 GWASs. To evaluate if the select

718 ion of $\sim 1.7$ million SNPs biased the results, we compared distribution of these analysed SNPs

719 with the all known SNPs in the genome (SNPs exist in 1000G EUR population, UKB1 and

720 UKB2 reference panels) by computing the proportion of SNPs per chromosome. In addition,

721 distribution of functional consequences of SNPs annotated by ANNOVAR ${ }^{36}$ was also compared with the all SNPs in the genome. For each SNP, we counted the number of traits to which a SNP was significantly associated at $p<5 \mathrm{e}-8$, and then grouped the associated SNPs into multi-domain, domain-specific and trait-specific SNPs using the same definitions as at the gene level.

726 Functional consequences of SNPs were annotated using ANNOVAR ${ }^{36}$. To test if a SNP from a certain functional category is enriched at a given number of associated domains compared to all analysed SNPs, a baseline proportion was calculated from the 1,740,179 SNPs for each functional category. At each number of associated domains (from 1 to 10 or more domains), we re-calculated the proportion of SNPs with each functional category and performed the

731 Fisher's exact test (one-sided) against the baseline (the proportion relative to all 1,740,179 SNPs), to test if the proportion if higher than expected. eQTLs for 48 tissue types were obtained from GTEx v7 (https://www.gtexportal.org/home/; last accessed 20 January 2018$)^{20}$ and we considered SNPs with gene q-value $<0.05$ with any gene in any tissue as eQTLs. For each eQTL, we counted the number of tissue types of being eQTL (regardless of associated genes) and categorized them into five groups, i.e. being eQTLs in $i$ ) a single tissue type (tissue specific eQTLs), $i$ ) between two and 12, iii) between 13 and $24, i x$ ) between 25 and 36 and $x$ ) and being in more than 37 tissue types. At each number of associated domains, we re-calculated the proportion of SNPs in each of the 5 categories, and performed the Fisher's exact test (one-sided) against baseline (the proportion relative to all $1,740,179 \mathrm{SNPs})$, to test if the proportion if higher than expected. 


\section{Pleiotropic gene-sets}

744 For gene-set level pleiotropy, we extracted 10,650 gene-sets tested in all 588 traits. We then considered gene-sets with $p<4.69 \mathrm{e}-6(0.05 / 10,650)$ as significantly associated. The trait associated gene-sets were grouped into multi-domain, domain-specific and trait-specific gene-sets with the same definitions as at the gene level.

We compared the number of genes and average gene-length across gene-sets in different association categories and non-associated genes. Gene length was based on the start and end position of genes extracted from R package, biomaRt. We performed two-sided t-test in log scale of the number of genes and average gene-length.

752

\section{Power calculation of genetic correlation}

754 Power calculations were performed using the bivariate analysis of GCTA-GRML power calculator (http://cnsgenomics.com/shiny/gctaPower/) ${ }^{37}$, to estimate the minimum $r_{g}$ that obtain a power of 0.8 in the worst case scenario. From 558 traits, two traits with the worst case scenarios were selected, one with the minimum $h^{2}{ }_{S N P}$ estimated by LDSC and another with the minimum sample size. For each case, we obtained the minimum $r_{g}$ to obtain power of 0.8 by assuming both traits are quantitative with same sample size and $h^{2}$ SNP and have phenotypic correlation 0.1 .

\section{Hierarchical clustering of trait based on genetic correlation}

763 Hierarchical clustering was performed on the matrix of pair-wise $r_{g}$ 's as calculated between

764 the 558 traits. After Bonferroni correction for all possible trait pairs, non-significant genetic 765 correlations were replaced with 0 . The number of clusters $k$ was optimized between 50 and 766250 by maximizing the silhouette score with 30 iterations for each $k$. 


\section{Estimated standardized effect size of lead SNPs}

769

To enable comparison of effect sizes across GWASs from different studies, we first

converted P-values into Z-statistics (two sided) and expressed the estimated effect size as a

function of MAF and sample size as described previously ${ }^{21}$ using the following equations:

772

$$
\hat{b}=\frac{z}{\sqrt{2 p(1-p)\left(n+z^{2}\right)}}, \quad S E=\frac{1}{\sqrt{2 p(1-p)\left(n+z^{2}\right)}}
$$

773

where $p$ is MAF and $\mathrm{n}$ is the total sample size. We used the MAF of a corresponding

European reference panel (either 1000G, UKB1 or UKB2) as described in the previous interested in the direction of effect, we used squared standardized effect sizes for analyses in this study.

778

\section{Fine-mapping of trait-associated loci}

We defined the region to fine-map by taking $50 \mathrm{~kb}$ around the top SNPs of the trait-associated loci. When trait-associated loci were larger than the $50 \mathrm{~kb}$ window, the largest boundary was taken. Due to the complex LD structure, loci overlapping with the MHC region (chr6:25Mb$36 \mathrm{Mb}$ ) were excluded. The fine-mapping was performed using the FINEMAP software (http://www.christianbenner.com/\#) with shotgun stochastic search algorithm ${ }^{25}$. Since the coverage of true causal SNPs is affected by the sample size of the reference panel and $\mathrm{GWASs}^{38}$, we used randomly selected unrelated 100k EUR individuals from UKB2 cohort for all 558 GWASs. We limited the number of maximum causal SNPs $(k)$ per locus to 10. When the number of SNPs within a locus is relatively small (around 30 or less), the algorithm can fail to converge. In that case, $\mathrm{k}$ was decreased by 1 until FINEMAP was successfully run. 
FINEMAP outputs a set of models (all possible combination of $k$ causal SNPs in a locus) credible set SNPs were defined as unique SNPs included in the $95 \%$ credible set of models.

For each SNP, a posterior inclusion probability (PIP) was calculated as the sum of PPs of all models that contains that SNP. To select most likely causal SNPs, we further defined credible

\section{Information 9.}

\section{Annotation and characterization of lead SNPs and credible SNPs}

801 Functional consequences of SNPs were annotated using ANNOVAR ${ }^{36}$ based on Ensembl gene annotations on hg19. Prior to ANNOVAR, we aligned the ancestral allele with dbSNP

804 (http://egg2.wustl.edu/roadmap/data/byFileType/chromhmmSegmentations/ChmmModels/co

805 reMarks/jointModel/final/all.mnemonics.bedFiles.tgz; last accessed 16 Mar 2016) and we annotated one of the 15-core chromatin states to each of the lead SNPs based on chromosome coordinates. Subsequently, consequence state was assigned for each SNP by taking the most common state across 127 cell/tissue types. SNPs with consequence state $\leq 7$ were defined as

809 active. eQTLs in 48 tissue types were obtained from GTEx v $7^{20}$ and we only used the

810 significant eQTLs at gene q-value $<0.05$. eQTLs were assigned to SNPs by matching

811 chromosome coordinate and alleles.

812 As we showed that trait-associated loci have higher gene density compared to non-associated

813 regions, and GWAS signals are known to be enriched in regulatory elements ${ }^{40}$, we first

814 identified background enrichment by comparing SNPs within trait-associated loci or fine- 
combining all SNPs in 1000G, UKB1 and UKB2 reference panels ( 28 million SNPs in

817 total). SNPs within the trait-associated loci were defined as the ones with $\mathrm{P}$-value $<0.05$ and

$818 r^{2}>0.6$ with one of the independent significant SNPs as described above (see section

819 'Definition of lead SNPs and trait-associated loci'). Therefore, it does not necessary include

820 all SNPs physically located within the trait-associated loci. On the other hand, SNPs within

821 fine-mapped region include all SNPs physically located within 50kb window from the most significant SNP of a locus. To characterize lead SNPs and credible SNPs given background enrichments, we compared these SNPs against all SNPs within trait-associated loci or finemapped regions, respectively.

SNP heritability estimation with SumHer using LDAK model

We estimated SNP heritability of 558 traits using the SumHer function from the LDAK software v5.0 (http://dougspeed.com/ldak/) ${ }^{27}$. Since our purpose was to compare estimates from LDSC and SumHer, we used the 1000G EUR reference panel and extracted HapMap3

830 SNPs as consistent with LDSC. We used unique ID's of SNPs (consisting of chromosome:posision:allele 1:allele2) instead of rsID to maximize the match between

832 GWAS summary statistics and the reference panel. The MHC region (chr6:25Mb-34Mb) was excluded. As recommended by the author, SNPs with large effects $\left(Z^{2} /\left(Z^{2}+n\right)>100\right.$ where $Z^{2}$

834 is chi-squared statistics and $n$ is sample size of the SNP) were excluded.

835 To obtain SNP heritability in a liability scale, we provided population prevalence and sample 836 prevalence with flags '--prevalance' and '--ascertainment' for binary traits. The same 837 population prevalence was used as described in the section of SNP heritability estimate with

838 LDSC (Supplementary Table 25). Details results are discussed in Supplementary

\section{Information 10}


841 Estimation of polygenicity and discoverability with MiXeR

842 In the causal mixture model for GWAS summary statistics (MiXeR) proposed by Holland et

843 al., the distribution of SNP effect sizes is treated a mixture of two distributions for causal and

844 non-causal SNPs as the following ${ }^{28}$ :

$$
\beta=\pi N\left(0, \sigma_{\beta}^{2}\right)+(1-\pi) N(0,0)
$$

846 where $\pi$ is the proportion of (independent) causal SNPs and $\sigma_{\beta}{ }^{2}$ is the variance of the effect

847 sizes of causal SNPs. Therefore, $\pi$ and $\sigma_{\beta}{ }^{2}$ respectively represent polygenicity and

848 discoverability of the trait. We estimated both parameters for the 558 traits using MiXeR

849 software (https://github.com/precimed/mixer) ${ }^{28}$. As recommended in the original study, we

850 used $1000 \mathrm{G}$ EUR as a reference panel and restricted to HapMap 3 SNPs. SNPs with $\chi^{2}>80$

851 and the MHC region (chr6:26Mb-34Mb) were excluded. To estimate the sample size required

852 to explain $90 \%$ of the additive genetic variance of a phenotype, we used an output of GWAS

853 power estimates calculated in the MiXeR software, which contains 51 data points of sample

854 size and the proportion of chip heritability explained ${ }^{28}$. We then estimated the sample size

855 required to reaches $90 \%$ by using the interpl function from the pracma package in R.

856

857 Data and materials availability

858 All publicly available GWAS summary statistics (original) files curated in this study are

859 accessible from the original links provided at http://atlas.ctglab.nl. GWAS summary statistics

860 for 600 traits from UK Biobank performed in this study are also provided at

861 http://atlas.ctglab.nl and an archived file will be made available upon publication from

862 https://ctg.cncr.nl/software/summary_statistics. 


\section{REFERENCES}

865

866

867

868

869

870

871

872

873

874

875

876

877

878

879

880

881

882

883

884

885

886

887

888

889

890

891

892

893

894

895

896

897

898

899

900

901

902

903

904

905

906

907

908

909

910

911

1. Edwards, A. O. et al. Complement factor H polymorphism and age-related macular degeneration. Science (80-. ). 308, 421-425 (2005).

2. Welter, D. et al. The NHGRI GWAS Catalog, a curated resource of SNP-trait associations. Nucleic Acids Res. 42, D1001-D1006 (2014).

3. Lander, E. S. Initial impact of the sequencing of the human genome. Nature 470, 187197 (2011).

4. Visscher, P. M. et al. 10 Years of GWAS Discovery : Biology, Function, and Translation. Am. J. Hum. Genet. 101, 5-22 (2017).

5. Henderson, P. \& Stevens, C. The role of autophagy in Crohn's Disease. Cells 1, 492519 (2012).

6. Okada, Y. et al. Genetics of rheumatoid arthritis contributes to biology and drug discovery. Nature 506, 376-81 (2014).

7. Gaulton, K. J. et al. Genetic fine mapping and genomic annotation defines causal mechanisms at type 2 diabetes susceptibility loci. Nat. Genet. 47, 1415-1425 (2015).

8. Canela-Xandri, O., Rawlik, K. \& Tenesa, A. An atlas of genetic associations in UK Biobank. Nat. Genet. 50, 1593-1599 (2018).

9. Timpson, N. J., Greenwood, C. M. T., Soranzo, N., Lawson, D. J. \& Richards, J. B. Genetic architecture: The shape of the genetic contribution to human traits and disease. Nat. Rev. Genet. 19, 110-124 (2018).

10. Boyle, E. A., Li, Y. I. \& Pritchard, J. K. An expanded view of complex traits: from polygenic to omnigenic. Cell 169, 1177-1186 (2017).

11. Wray, N. R., Wijmenga, C., Sullivan, P. F., Yang, J. \& Visscher, P. M. Common disease is more complex than implied by the core gene omnigenic model. Cell 173, 1573-1580 (2018).

12. Bycroft, C. et al. The UK Biobank resource with deep phenotyping and genomic data. Nature 562, 203-209 (2018).

13. Goh, K. et al. The human disease network. Proc. Natl. Acad. Sci. 104, 8685-8690 (2007).

14. Polderman, T. J. C. et al. Meta-analysis of the heritability of human traits based on fifty years of twin studies. Nat. Publ. Gr. 47, 702-709 (2015).

15. Mahajan, A. et al. Fine-mapping type 2 diabetes loci to single-variant resolution using high-density imputation and islet-specific epigenome maps. Nat. Genet. (2018).

16. de Leeuw, C. A., Mooij, J. M., Heskes, T. \& Posthuma, D. MAGMA: generalized gene-set analysis of GWAS data. PLoS Comput. Biol. 11, e1004219 (2015).

17. Bulik-sullivan, B. K. et al. LD Score regression distinguishes confounding from polygenicity in genome-wide association studies. Nat. Genet. 47, 291-295 (2015).

18. Solovieff, N., Cotsapas, C., Lee, P. H., Purcell, S. M. \& Smoller, J. W. Pleiotropy in complex traits: Challenges and strategies. Nat. Rev. Genet. 14, 483-495 (2013).

19. Lek, M. et al. Analysis of protein-coding genetic variation in 60,706 humans. Nature 536, 285-291 (2016).

20. The GTEx Consortium. Genetic effects on gene expression across human tissues. Nature 550, 204-213 (2017).

21. Zhu, Z. et al. Integration of summary data from GWAS and eQTL studies predicts complex trait gene targets. Nat. Genet. 48, 481-487 (2016).

22. Manolio, T. A. et al. Finding the missing heritability of complex diseases. Nature 461, 747-753 (2009).

23. Lee, S., Abecasis, G. R., Boehnke, M. \& Lin, X. Rare-variant association analysis: 
Study designs and statistical tests. Am. J. Hum. Genet. 95, 5-23 (2014).

24. van de Bunt, M., Cortes, A., Brown, M. A., Morris, A. P. \& McCarthy, M. I. Evaluating the performance of fine-mapping strategies at common variant GWAS loci. PLoS Genet. 11, e1005535 (2015).

25. Benner, C. et al. FINEMAP : efficient variable selection using summary data from genome-wide association studies. Bioinformatics 32, 1493-1501 (2016).

26. Speed, D. et al. Reevaluation of SNP heritability in complex human traits. Nat. Genet. 49, 986-992 (2017).

27. Speed, D. \& Balding, D. J. Better estimation of SNP heritability from summary statistics provides a new understanding of the genetic architecture of complex traits. Nat. Genet. (2018).

28. Holland, D. et al. Beyond SNP heritability: polygenicity and discoverability estimated for multiple phenotypes with a univariate gaussian mixture model. bioRxiv (2018).

29. Purcell, S. et al. PLINK: A tool set for whole-genome association and populationbased linkage analyses. Am. J. Hum. Genet. 81, 559-575 (2007).

30. Watanabe, K., Taskesen, E., van Bochoven, A. \& Posthuma, D. Functional mapping and annotation of genetic associations with FUMA. Nat. Commun. 8, 1826 (2017).

31. Auton, A. et al. A global reference for human genetic variation. Nature 526, 68-74 (2015).

32. Liberzon, A. et al. Molecular signatures database (MSigDB) 3.0. Bioinformatics 27, 1739-1740 (2011).

33. ZHENG, J. et al. LD Hub: a centralized database and web interface to perform LD score regression that maximizes the potential of summary level GWAS data for SNP heritability and genetic correlation analysis. Bioinformatics 33, 272-279 (2017).

34. Yengo, L. et al. Meta-analysis of genome-wide association studies for height and body mass index in 700000 individuals of European ancestry. Hum. Mol. Genet. 27, 36413649 (2018).

35. Giambartolomei, C. et al. Bayesian Test for Colocalisation between Pairs of Genetic Association Studies Using Summary Statistics. PLoS Genet. 10, e1004383 (2014).

36. Wang, K., Li, M. \& Hakonarson, H. ANNOVAR: functional annotation of genetic variants from high-throughput sequencing data. Nucleic Acids Res. 38, e164 (2010).

37. Visscher, P. M. et al. Statistical power to detect genetic (co)variance of complex traits using SNP data in unrelated samples. PLoS Genet. 10, e1004269 (2014).

38. Benner, C. et al. Prospects of fine-mapping trait-associated genomic regions by using summary statistics from genome-wide association studies. Am. J. Hum. Genet. 101, 539-551 (2017).

39. Roadmap Epigenomics Consortium. Integrative analysis of 111 reference human epigenomes. Nature 518, 317-330 (2015).

40. Tak, Y. G. \& Farnham, P. J. Making sense of GWAS: using epigenomics and genome engineering to understand the functional relevance of SNPs in non-coding regions of the human genome. Epigenetics Chromatin 8, 57 (2015). 


\section{END NOTES}

955 Acknowledgement We thank all consortiums and all other individual labs for making

956 GWAS summary statistics publicly available. We also thank Peter Visscher and Naomi Wray

957 for their thoughtful suggestions and discussions. We additionally thank Anders Dale for his

958 suggestions for the manuscript. This work was funded by Netherlands Organization for

959 Scientific Research (NWO VICI 453-14-005 and NWO VIDI 452-12-014).

960 Author contribution D.P. designed the study. K.W. curated the database and performed

961 analyses. T.J.C.P assisted with harmonization of phenotype labels of the database. S.S.

962 performed QC on the UK Biobank data and wrote the analysis pipeline for UKB analyses.

963 M.U.M assisted with the fine-mapping analyses. O.F. and O.A.A. developed software

964 UGMG and assisted with the analyses. S.v.d.S and B.M.N discussed and provided valuable

965 suggestions for analyses. K.W. and D.P. wrote the paper. All authors critically reviewed the

966 paper.

967 Competing interests The authors declare no competing financial interest.

968 Corresponding author Correspondence and requests for materials should be addressed to

969 D.P.(danielle.posthuma@vu.nl).

970 
971 Table 1. Count and proportion of pleiotropic trait-associated loci, genes, SNPs and

972 gene-sets.

\begin{tabular}{lrrrrrrr|rr}
\hline & \multicolumn{2}{c}{ Loci } & \multicolumn{4}{c}{ Genes } & \multicolumn{2}{c|}{ SNPs } & \multicolumn{2}{c}{ Gene-set } \\
\cline { 2 - 10 } & Length (Mb) & \multicolumn{1}{c}{$\%$} & Count & \% & Count & \% & Count & $\%$ \\
\hline Total in genome & 2796.10 & 100.00 & 17,444 & 100.00 & $1,740,179$ & 100.00 & 10,650 & 100.00 \\
\hline Associated & 1706.00 & 61.01 & 11,443 & 65.60 & 236,388 & 13.58 & 1,106 & 10.38 \\
Pleiotropic* & 1592.53 & 93.35 & 9,252 & 80.85 & 142,376 & 60.23 & 606 & 54.79 \\
Multi-domain & 1535.76 & 90.02 & 7,657 & 66.91 & 76,650 & 32.43 & 361 & 32.64 \\
Domain specific & 56.77 & 3.33 & 1,595 & 13.94 & 65,726 & 27.80 & 245 & 22.15 \\
Trait specific & 113.48 & 6.65 & 2,191 & 19.15 & 94,012 & 39.77 & 500 & 45.21 \\
\hline Non-associated & 1090.10 & 38.99 & 6,001 & 34.40 & $1,503,791$ & 86.42 & 9,544 & 89.61 \\
\hline
\end{tabular}

973 *The count of pleiotropic loci, genes, SNPs and gene-sets is the sum of the multi-domain and

974 domain specific categories. Proportion of pleiotropic, multi-domain, domain specific and trait

975 specific categories are relative to the associated loci, SNPs, genes or gene-sets, respectively. 
Table 2. Characteristics of lead SNPs and credible SNPs with PIP>0.95 across 558 traits versus all SNPs in the genome.

\begin{tabular}{|c|c|c|c|c|c|c|c|c|c|c|c|c|c|}
\hline \multirow{2}{*}{$\begin{array}{c}\text { Annotation } \\
\text { categories }\end{array}$} & \multirow{2}{*}{\begin{tabular}{c|} 
Genome \\
$\%$
\end{tabular}} & \multicolumn{3}{|c|}{ Trait-associated loci } & \multicolumn{3}{|c|}{ lead SNPs } & \multicolumn{3}{|c|}{$50 \mathrm{~kb}$ around the top SNPs ${ }^{\mathrm{a}}$} & \multicolumn{3}{|c|}{ Credible SNPs (PIP $>0.95)^{b}$} \\
\hline & & $\%$ & $\mathbf{E}$ & $\mathbf{P}^{\mathbf{c}}$ & $\%$ & $\mathbf{E}$ & $\mathbf{P}^{\mathbf{d}}$ & $\%$ & $\mathbf{E}$ & $\mathbf{P}^{\mathbf{c}}$ & $\%$ & $\mathbf{E}$ & $\mathbf{P}^{\mathbf{e}}$ \\
\hline Non-coding & 94.37 & 93.06 & 0.99 & $<1 \mathrm{e}-323 !$ & 89.13 & 0.96 & $1.14 \mathrm{E}-185$ & 94.04 & 1.00 & $<1 \mathrm{e}-323 !$ & 92.39 & 0.98 & $7.60 \mathrm{E}-192$ \\
\hline Intergenic & 44.11 & 36.88 & 0.84 & $<1 \mathrm{e}-323$ & 34.31 & 0.93 & $1.20 \mathrm{E}-27$ & 41.41 & 0.94 & $<1 \mathrm{e}-323$ & 33.40 & 0.81 & $<1 \mathrm{e}-323$ \\
\hline Intronic & 38.29 & 44.88 & 1.17 & $<1 \mathrm{e}-323$ & 43.85 & 0.98 & $2.38 \mathrm{E}-05$ & 41.14 & 1.07 & $<1 \mathrm{e}-323$ & 48.07 & 1.17 & $<1 \mathrm{e}-323$ \\
\hline scRNA intronic & 11.98 & 11.29 & 0.94 & $1.34 \mathrm{E}-115$ & 10.98 & 0.97 & 0.044458 & 11.49 & 0.96 & $<1 \mathrm{e}-323$ & 10.92 & 0.95 & $2.49 \mathrm{E}-15$ \\
\hline Coding & 2.15 & 2.40 & 1.12 & $7.42 \mathrm{E}-73$ & 4.60 & 1.92 & $1.33 \mathrm{E}-147$ & 2.27 & 1.06 & $4.02 \mathrm{E}-186$ & 2.86 & 1.26 & $7.38 \mathrm{E}-63$ \\
\hline Exonic & 1.06 & 1.13 & 1.07 & $2.27 \mathrm{E}-14$ & 3.22 & 2.84 & $1.30 \mathrm{E}-230$ & 1.20 & 1.14 & $<1 \mathrm{e}-323$ & 1.68 & 1.40 & $1.62 \mathrm{E}-73$ \\
\hline Splicing & $1.16 \mathrm{E}-02$ & $1.13 \mathrm{E}-02$ & 0.98 & 8.62E-01: & $2.11 \mathrm{E}-02$ & 1.86 & 0.102234 & $1.29 \mathrm{E}-02$ & 1.11 & $7.00 \mathrm{E}-05$ & $1.95 \mathrm{E}-02$ & 1.51 & $1.59 \mathrm{E}-02$ \\
\hline ncRNA exonic & 1.07 & 1.25 & 1.16 & $6.02 \mathrm{E}-71 !$ & 1.36 & 1.09 & 0.04846 & 1.05 & 0.98 & 5.12E-11 & 1.16 & 1.10 & 4.14E-06 \\
\hline ncRNA splicing & $5.40 \mathrm{E}-03$ & $5.09 \mathrm{E}-03$ & 0.94 & $7.03 \mathrm{E}-01$ & $2.35 \mathrm{E}-03$ & 0.46 & 0.72602 & $5.25 \mathrm{E}-03$ & 0.97 & $5.06 \mathrm{E}-01$ & $3.07 \mathrm{E}-03$ & 0.59 & $2.66 \mathrm{E}-01$ \\
\hline Flanking regions & 3.48 & 4.54 & 1.31 & $<1 \mathrm{e}-323$ & 6.27 & 1.38 & $4.60 \mathrm{E}-57$ & 3.68 & 1.06 & $1.04 \mathrm{E}-299$ & 4.75 & 1.29 & $1.48 \mathrm{E}-125$ \\
\hline Upstream & 1.09 & 1.33 & 1.22 & $9.09 \mathrm{E}-124$ & 1.64 & 1.23 & $1.08 \mathrm{E}-07$ & 1.09 & 1.00 & 7.59E-01! & 1.29 & 1.18 & $5.45 \mathrm{E}-16$ \\
\hline 5' UTR & 0.30 & 0.44 & 1.48 & 4.61E-151! & 0.78 & 1.76 & $1.64 \mathrm{E}-20$ & 0.35 & 1.16 & 4.71E-183! & 0.57 & 1.66 & $8.75 \mathrm{E}-55$ \\
\hline 3' UTR & 0.98 & 1.32 & 1.34 & $2.41 \mathrm{E}-260$ & 2.06 & 1.56 & $5.69 \mathrm{E}-34$ & 1.13 & 1.15 & $<1 \mathrm{e}-323$ & 1.67 & 1.48 & $2.47 \mathrm{E}-98$ \\
\hline Downstream & 1.10 & 1.45 & 1.32 & $4.18 \mathrm{E}-256$ & 1.79 & 1.23 & $3.38 \mathrm{E}-08$ & 1.11 & 1.01 & $9.73 \mathrm{E}-03$ & 1.21 & 1.09 & 5.23E-05 \\
\hline Active chromatin & 17.24 & 27.74 & 1.61 & $<1 \mathrm{e}-323$ & 30.10 & 1.08 & $1.24 \mathrm{E}-27$ & 20.63 & 1.20 & $<1 \mathrm{e}-323$ & 31.06 & 1.51 & $<1 \mathrm{e}-323$ \\
\hline eQTLs & 9.66 & 57.41 & 5.95 & $<1 \mathrm{e}-323$ & 46.15 & 0.80 & $7.54 \mathrm{E}-190$ & 11.45 & 1.19 & $<1 \mathrm{e}-323$ & 47.47 & 4.14 & $<1 \mathrm{e}-323$ \\
\hline
\end{tabular}

978 E: fold enrichment (proportion of SNPs with a certain annotation divided by the proportion of SNPs with the same annotation in background).

979 anly including the fine-mapped regions (for loci larger than $50 \mathrm{~kb}$ windows from the top SNPs, the largest boundaries were taken). ${ }^{b}$ From $95 \%$

980 credible set SNPs, only SNPs with posterior inclusion probability (PIP) $>0.95$ were selected. cP-value of Fisher's exact test (two-sided) against

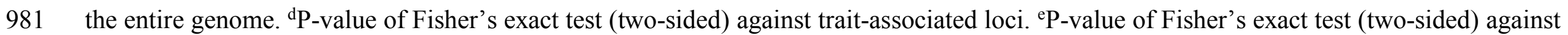

$98250 \mathrm{~kb}$ around the top SNPs 

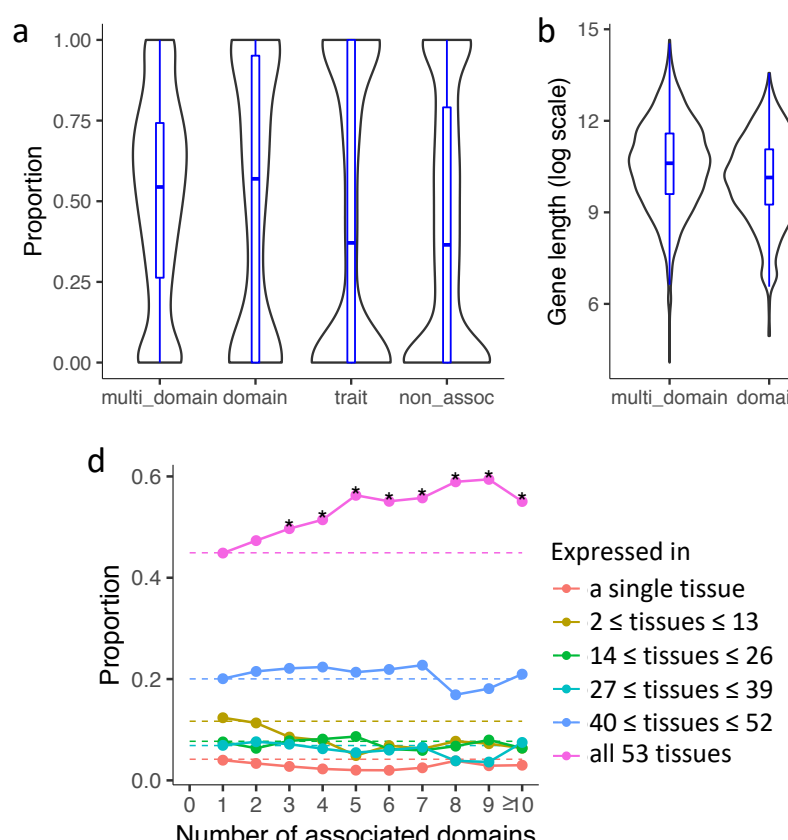

$\mathrm{f}$

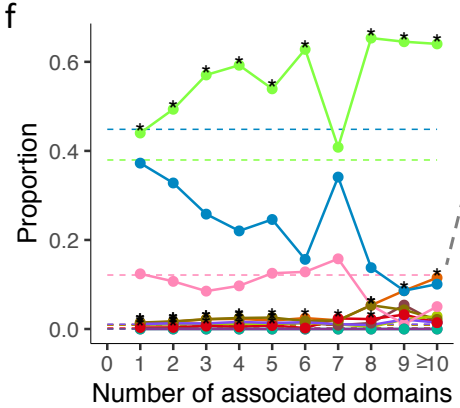

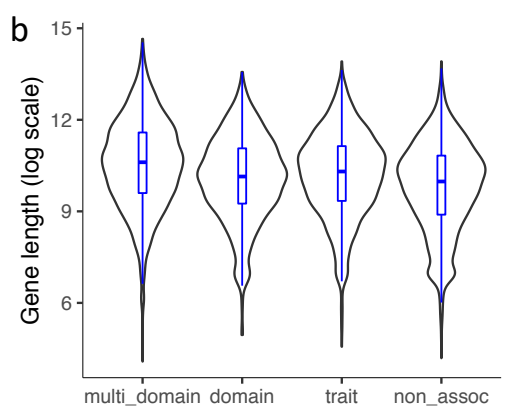
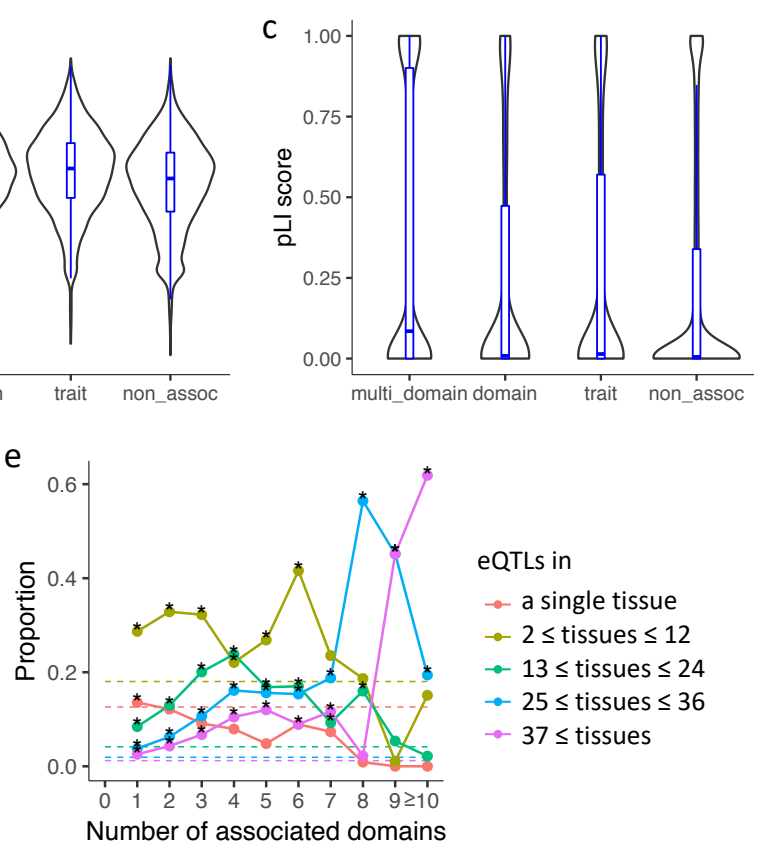

eQTLs in

$\rightarrow$ a single tissue

$\rightarrow 2 \leq$ tissues $\leq 12$

$\rightarrow 13 \leq$ tissues $\leq 24$

$\rightarrow 25 \leq$ tissues $\leq 36$

$\rightarrow 37 \leq$ tissues

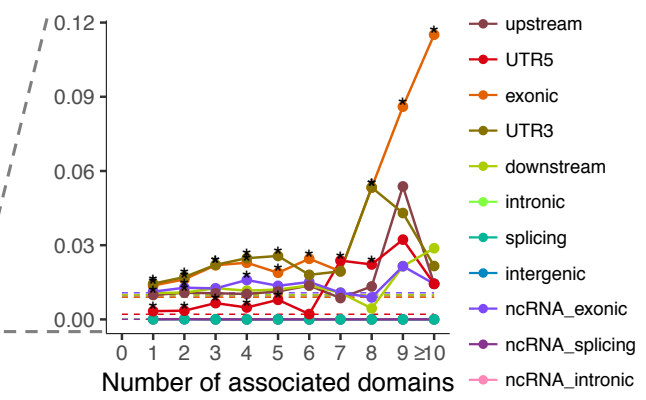

Fig. 1. Trait-associated locus, gene and SNP pleiotropy across the genome. a.

985 Distribution of gene density of loci with different association types. b. Distribution of gene

986 length in log scale with different association types. c. Distribution of pLI score of genes with

987 different association types. For a-c, multi_domain: associated with traits from $>1$ domain,

988 domain: associated with $>1$ traits from a single domain, trait: associated with a single trait,

989 non_assoc: not associated with any of 558 traits. d. Tissue specificity of genes at different

990 levels of pleiotropy. Each data point represents a proportion of genes expressed in a given

991 number of tissues for a specific number of associated domains. e. Proportion of SNPs with

992 different functional consequences at different levels of pleiotropy. Each data point represents

993 the proportion of SNPs with a given functional consequence for a specific number of 
994 associated domains. f. Tissue specificity of SNPs based on active eQTLs at different levels of 995 pleiotropy. Each data point represents the proportion of SNPs being eQTLs in a given

996 number of tissues for a specific number of associated domains. For d-f, dashed lines refer to

997 the baseline proportions (relative to all 17,444 genes (d) or all 1,740,179 SNPs (e-f)), and

998 stars denote significant enrichment relative to the baseline (Fisher's exact test, one-sided).

999 

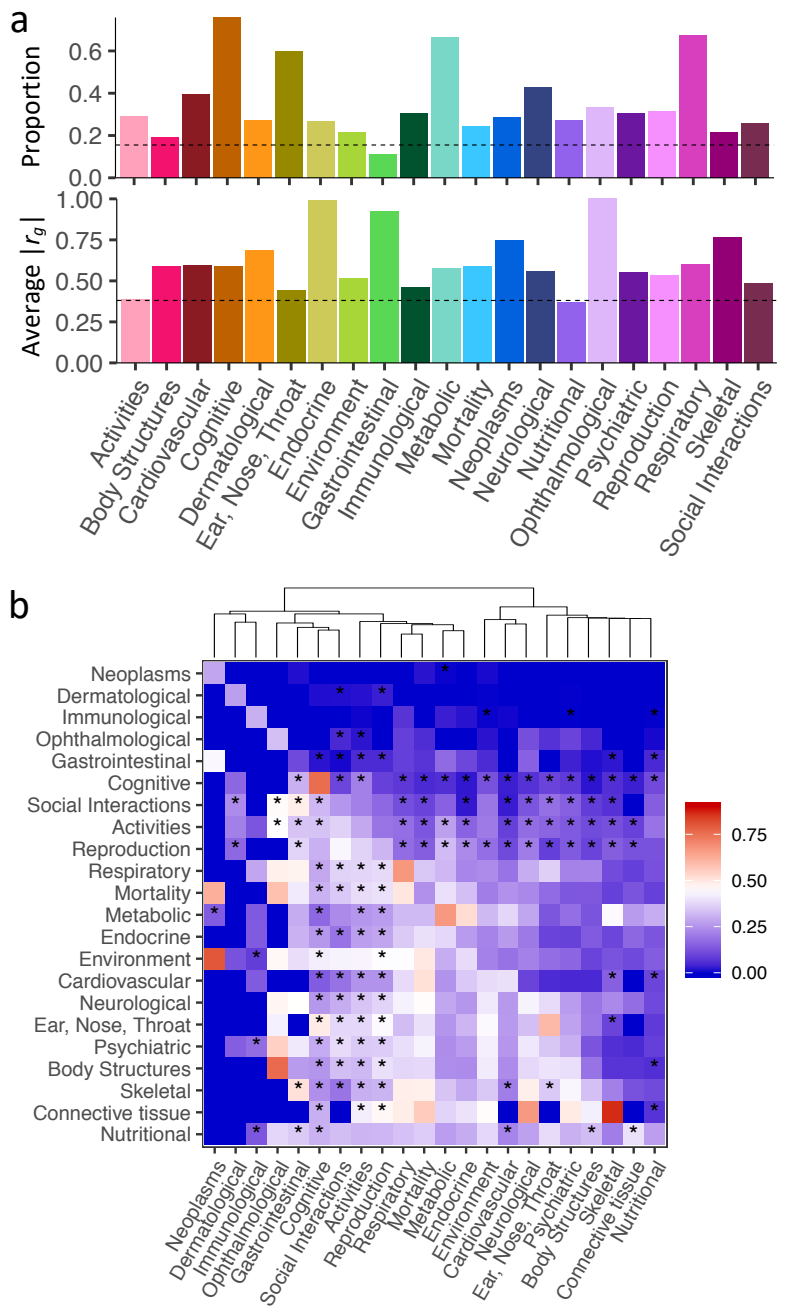

1001 Fig. 2. Within and between domains genetic correlations. a. Proportion of trait pairs with

1002 significant $r_{g}$ (top) and average $\left|r_{g}\right|$ for significant trait pairs (bottom) within domains. Dashed

1003 lines represent the proportion of trait pairs with significant $r_{g}$ (top) and average $\left|r_{g}\right|$ for

1004 significant trait pairs (bottom) across all 558 traits, respectively. Connective tissue, muscular 1005 and infection domains are excluded as these each contains less than 3 traits. b. Heatmap of

1006 proportion of trait pairs with significant $r_{g}$ (upper right triangle) and average $\left|r_{g}\right|$ for

1007 significant trait pairs (lower left triangle) between domains. Connective tissue, muscular and

1008 infection domains are excluded as each contains less than 3 traits. The diagonal represents the

1009 proportion of trait pairs with significant $r_{g}$ within domains. Stars denote the pairs of domains

1010 in which the majority ( $>50 \%$ ) of significant $r_{g}$ are negative. 
a

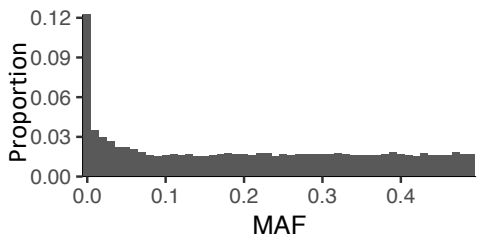

b

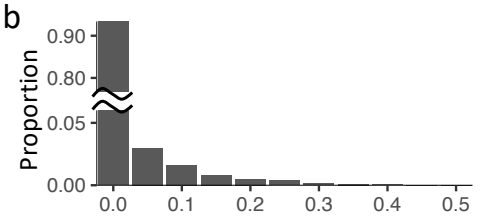

Squared standardized effect size

d

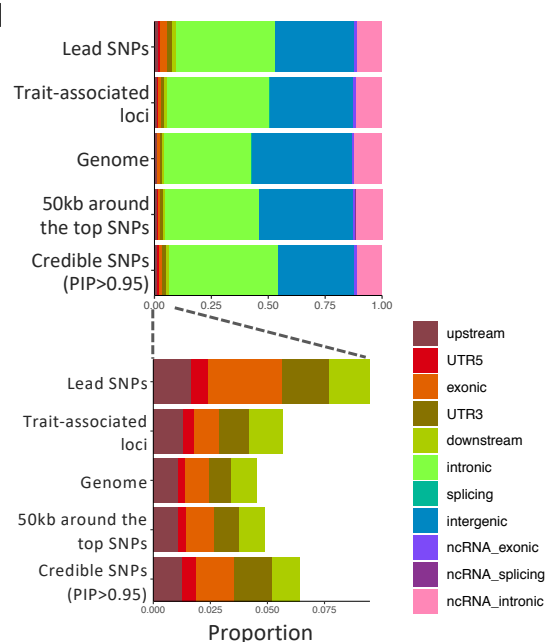

C

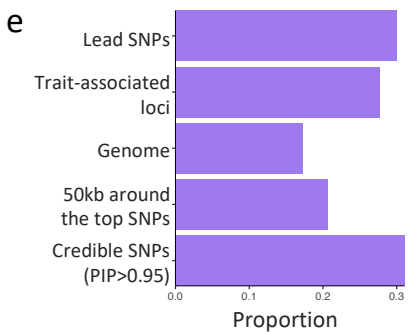

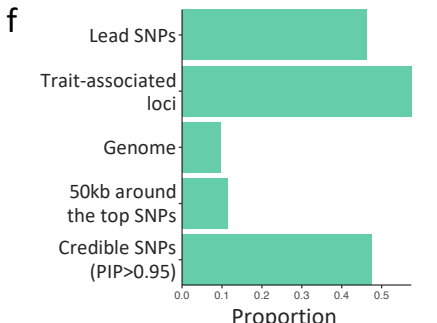

1011

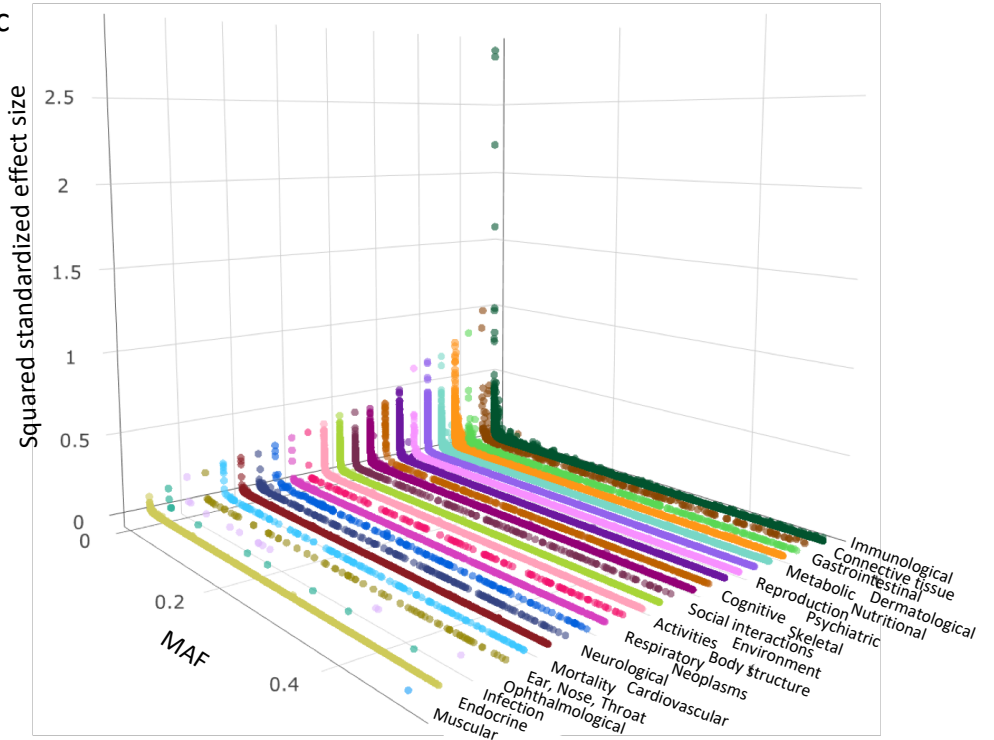

1012 Fig. 3. Distribution and characterization of lead SNPs and credible SNPs of 558 traits. a.

1013 Histogram of MAF of the unique lead SNPs. b. Histogram of squared standardized effect size

1014 of lead SNPs. c. Scatter plot of MAF and squared standardized effect sizes of lead SNPs

1015 grouped by trait domains. d. Distribution of functional consequences of SNPs. e. Proportion

1016 of SNPs that overlap with active consequence chromatin state $(\leq 7)$ across 127 tissue/cell

1017 types. f. Proportion of SNPs overlapping with significant eQTLs from any of 48 available

1018 tissue types.

1019 


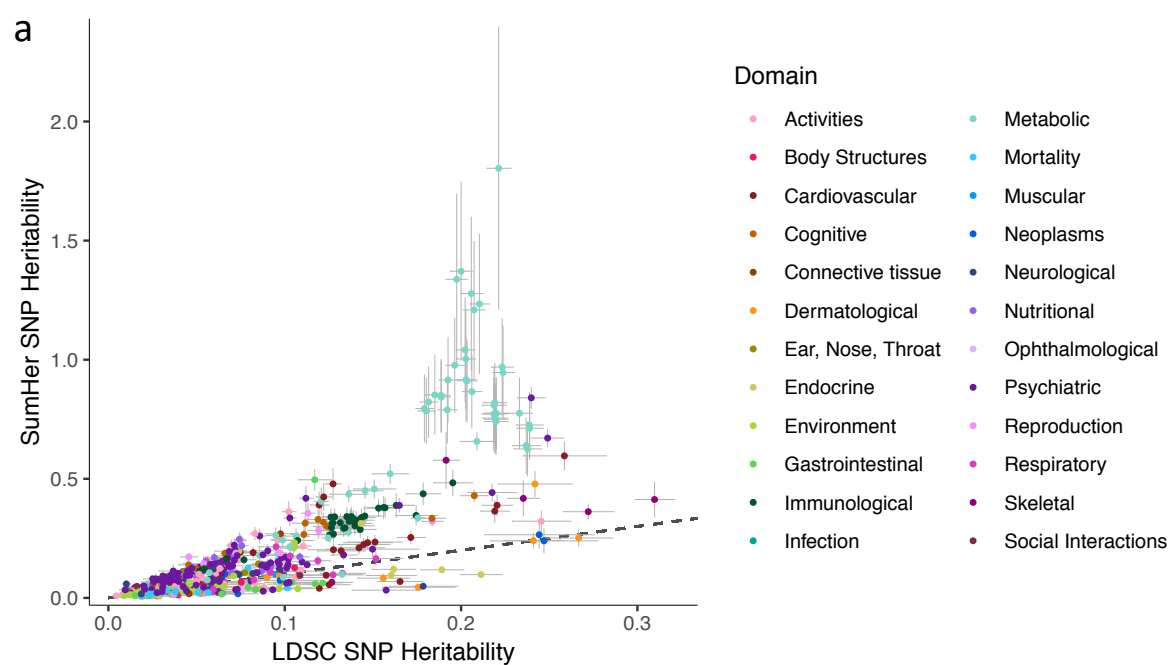

1020
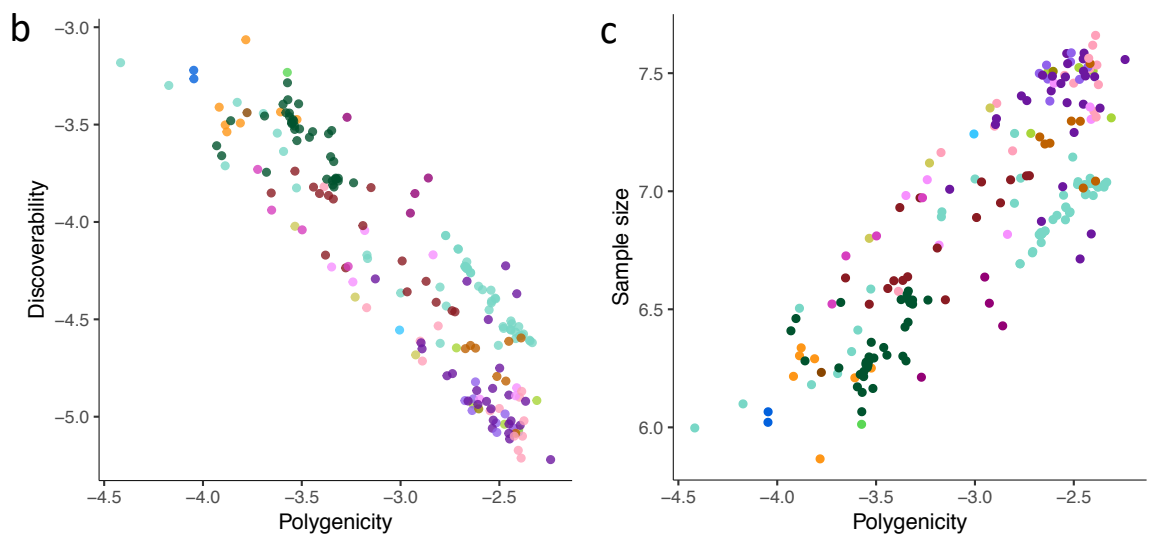

1021 Fig. 4. SNP heritability and polygenicity of 558 traits. a. Comparison of SNP heritability estimated by LDSC (x-axis) and SumHer (y-axis). Horizontal and vertical error bar represent standard errors of LDSC and SumHer estimates, respectively. b. Polygenicity and

1024 discoverability of traits, both on $\log 10$ scale. Out of 558 traits, only 197 traits with reliable 1025 estimates (i.e. $h^{2}{ }_{S N P}>0.05$ (estimated by MiXeR) and standard error of $\pi$ is less than $50 \%$ of 1026 the estimated value) are displayed. Traits are colored by domain. c. Polygenicity and estimated sample size required to reach $90 \%$ of total SNP heritability explained by genomewide significant SNPs, both in log 10 scale. Traits are colored by domain. Full results are available in Supplementary Table 22, 23. 